\title{
Incoherent single pion electroproduction on the deuteron with polarization effects
}

\author{
M. Tammam ${ }^{a}$, A. Fix ${ }^{b}$ and H. Arenhövel ${ }^{b}$ \\ ${ }^{a}$ Physics Department, Al-Azhar University, Asiut, Egypt, \\ ${ }^{b}$ Institut für Kernphysik, Johannes Gutenberg-Universität Mainz, D-55099 Mainz, Germany
}

(Dated: May 26, 2018)

\begin{abstract}
Incoherent pion electroproduction on the deuteron is studied from threshold up to the second resonance region with special emphasis on the influence of final state interaction, in particular on polarization observables. The elementary $\gamma N \rightarrow \pi N$ amplitude is taken from the MAID-2003 model. Final state interaction is included by considering complete rescattering in the final $N N$ and $\pi N$ subsystems. Their influence on the structure functions governing the semi-exclusive differential cross section, where besides the scattered electron only the produced pion is detected, is investigated in detail. For charged pion-production the effect of $N N$-rescattering is moderate whereas $\pi N$-rescattering is almost negligible, except very close to threshold. $N N$-rescattering appears much stronger in neutral pion production for which the primary mechanism is the elimination of a significant spurious coherent contribution in the impulse approximation. Sizeable effects are also found in some of the polarization structure functions for beam and/or target polarizations.
\end{abstract}

PACS numbers: 13.60.Le, 13.40.-f, 21.45.+v, 24.70+s, 25.30.Rw

\section{INTRODUCTION}

The present paper is an extension of previous work on electromagnetic single pion production on the deuteron 1, 2] in which we had considered the case of photoproduction. In the first part [1], a thorough derivation of the formal expressions for polarization observables in this reaction were presented. Then in [2] we had systematically investigated this process using as realistic elementary pion production operator the MAID-2003 model [3] and included complete rescattering in the two-body $N N$ - and $\pi N$-subsystems of the final state (FSI). Moderate influences of the latter on total and semi-exclusive differential cross sections were found in charged pion production, primarily from $N N$-rescattering while $\pi N$-rescattering remained small. Much larger effects were found in incoherent neutral pion production, which, however, originated predominantly from the elimination of a sizeable spurious coherent contribution to the incoherent process in the impulse approximation (IA) where any final state interaction is neglected.

In view of the interest in this reaction with respect to (i) extracting on the one hand information on the elementary production on the neutron in using the deuteron as an effective neutron target, and (ii) studying the influence of the spectator nucleon, i.e. medium effects, it appears natural to investigate the corresponding electroproduction reaction taking advantage of the possibility to vary energy and momentum transfer independently in the space like region. For example, it would be interesting to see whether some kinematic regions exist where $\pi N$-rescattering becomes more important. Indeed, several studies of the role of FSI and medium effects have already been undertaken in the past, both experimentally [4, 5, 6] as well as theoretically $7,[8,9]$.

An early experiment by C.N. Brown et al. [4] was designed to study the isoscalar-isovector interference of the elementary amplitude and the role of nuclear corrections by measuring on the one hand the ratio of $\pi^{-}$to $\pi^{+}$ production on the deuteron and on the other hand the ratio of $\pi^{+}$production on the deuteron to the one on hydrogen. The forward-angle production of charged pions on the deuteron was measured by R. Gilman et al. [5] in order to investigate possible influences of the spectator nucleon on the elementary production amplitude. For the cross section ratio of $\pi^{+}$production on the deuteron to the one on the proton they found a significant deviation from unity. Their conclusion was that there is evidence for a modification of the elementary pion production process in the nuclear medium. In a subsequent theoretical paper, Loucks et al. [7] obtained within a simple model for the elementary pion production operator this ratio in fair agreement with experiment without invoking medium modifications. The deviation from unity was traced back to the strong final state interaction in the ${ }^{1} S_{0}$-partial wave of the outgoing neutrons in which the ${ }^{1} S_{0}$-anti-bound state is the dominant feature for low energies. In addition a strong dependence of the differential cross section on the tensor polarization of an oriented deuteron was found which is a manifestation of the non-spherical character of the deuteron via its $D$-state component.

A similar motivation with respect to possible medium modifications was also behind a more recent experiment by D. Gaskell et al. [6] which was triggered by the observation that as long as the longitudinal current is dominated by the pion pole term one could explore the nuclear pion field. Results were presented for longitudinal charged pion production on ${ }^{1} \mathrm{H},{ }^{2} \mathrm{H}$, and ${ }^{3} \mathrm{He}$ targets. The data, however, did not support any significant modification of the elementary production process by the presence of the spectator nucleons. At about the same time, Hafidi and Lee 8 published a theoretical study using a dynamical model for the e.m. pion production operator, allowing besides final 
state rescattering also to include intermediate baryon-baryon interactions, i.e., two-body contributions to the e.m. interaction. However, the latter turned out to be almost negligible in the near threshold region. The same nearthreshold region was also considered in another more recent theoretical paper by Levchuck et al. 9] using a unitary transformation method and restricting the e.m. current to the lowest multipoles based on the Born contributions alone, i.e. leaving out the contribution of the $\Delta$-resonance. The results of $[8]$ were confirmed with respect to the influence of FSI near the quasi-free peak but not for low missing mass.

In the present work we would like to study more systematically incoherent electroproduction of single pions on the deuteron using a realistic elementary pion production operator with respect to the importance of final state interactions in different energy regions, from threshold through the $\Delta$-resonance up to the second resonance region. In particular, the role of polarization degrees of freedom will be explored. In the next section, we will briefly review the formal aspects of this reaction, especially the definition of polarization observables. The results will be presented and discussed in Sect. 4, and we will close with a summary and an outlook.

\section{FORMALISM}

The basic formalism for electromagnetic single pion production on the deuteron has been presented in detail for the case of photoproduction in 1]. Therefore, we review here only the most important ingredients with due extensions to electroproduction according to the additional contributions from charge and longitudinal current components.

\section{A. Kinematics}

The kinematics of pion electroproduction in the one-photon exchange approximation is very similar to photoproduction in replacing the real photon by a virtual one with longitudinal and transverse polarizations

$$
\gamma^{*}(q)+d\left(p_{d}\right) \rightarrow \pi\left(p_{\pi}\right)+N_{1}\left(p_{1}\right)+N_{2}\left(p_{2}\right)
$$

defining here the notation of the four-momenta of the participating particles, i.e., $q=\left(q_{0}, \vec{q}\right)$ for the virtual photon, $p_{d}=\left(E_{d}, \vec{p}_{d}\right)$ for the deuteron, $p_{\pi}=\left(E_{\pi}, \vec{p}_{\pi}\right)$ for the produced pion, and $p_{i}=\left(E_{i}, \vec{p}_{i}\right)$ for the outgoing nucleons $(i=1,2)$. The momentum of the virtual photon is determined by the four-momentum transfer in the scattering process, i.e. $q=k_{e}-k_{e^{\prime}}$ denoting by $k_{e}=\left(E_{e}, \vec{k}_{e}\right)$ and $k_{e^{\prime}}=\left(E_{e^{\prime}}, \vec{k}_{e^{\prime}}\right)$ the momenta of incoming and scattered electrons, respectively. The electron kinematics will be considered in the laboratory frame, while the evaluation of the reaction matrix will be done in the center-of-momentum frame (c.m.) of virtual photon and deuteron, i.e. all variables, which determine the reaction matrix, refer to the c.m. frame if not indicated specifically otherwise.

As independent variables for the description of the final state we choose in the c.m. frame the outgoing pion momentum $\vec{p}_{\pi}=\left(p_{\pi}, \theta_{\pi}, \phi_{\pi}\right)$ and the spherical angles $\Omega_{p}=\left(\theta_{p}, \phi_{p}\right)$ of the relative momentum $\vec{p}=\left(\vec{p}_{1}-\vec{p}_{2}\right) / 2=\left(p, \Omega_{p}\right)$ of the two outgoing nucleons having momenta $\vec{p}_{1}$ and $\vec{p}_{2}$. In conjunction with the momentum of the virtual photon, the energies $E_{i}$ and momenta of the outgoing nucleons are fixed, i.e.

$$
\begin{aligned}
& E_{1 / 2}=\frac{1}{2} E_{12} \mp \frac{\vec{p} \cdot \vec{p}_{\pi}}{E_{12}}=\frac{1}{2} E_{12} \mp \frac{p p_{\pi}}{E_{12}} \cos \theta_{p \pi}, \\
& \vec{p}_{1 / 2}=-\frac{1}{2} \vec{p}_{\pi} \pm \vec{p}
\end{aligned}
$$

with $\theta_{p \pi}$ as angle between $\vec{p}$ and $\vec{p}_{\pi}$ and $E_{12}=E_{1}+E_{2}=W-E_{\pi}$ as the total final $N N$ energy, where

$$
W=q_{0}+\sqrt{M_{d}^{2}+q^{2}}=\sqrt{\left(2 q_{0}^{l a b}+M_{d}\right) M_{d}-Q^{2}}
$$

denotes the invariant total mass, $M_{d}$ the deuteron mass, and $Q^{2}=q_{\mu}^{2}$. Furthermore, the square of the relative momentum is fixed by the independent variables and is given by

$$
p^{2}=\frac{E_{12}^{2}\left(E_{12}^{2}-p_{\pi}^{2}-4 M^{2}\right)}{4\left(E_{12}^{2}-p_{\pi}^{2} \cos ^{2} \theta_{p \pi}\right)},
$$

where the nucleon mass is denoted by $M$. The pion momentum is restricted to $0 \leq p_{\pi} \leq p_{\pi, \max }$, where the upper limit is given by

$$
p_{\pi, \max }=\frac{1}{2 W} \sqrt{\left(\left(W-m_{\pi}\right)^{2}-4 M^{2}\right)\left(\left(W+m_{\pi}\right)^{2}-4 M^{2}\right)} .
$$


Of special interest is the quasi-free kinematics which is defined by the condition that the spectator nucleon remains at rest in the lab system, i.e. its final momentum is given by $p_{s}^{l a b}=(M, \overrightarrow{0})$. In this case, the lab energy of the active final pion-nucleon system is given by

$$
E_{\pi N}^{q f, l a b}=\sqrt{m_{\pi}^{2}+\left(\vec{p}_{\pi}^{l a b}\right)^{2}}+\sqrt{M^{2}+\left(\vec{q}^{l a b}-\vec{p}_{\pi}^{l a b}\right)^{2}}=M_{d}-M+q_{0}^{l a b} .
$$

For the semi-exclusive reaction, where besides the scattered electron only the produced pion is measured, one can determine the quasi-free lab pion energy $E_{\pi}^{q f, l a b}$ from (7) and finds

$$
\begin{aligned}
E_{\pi}^{q f, l a b}\left(\theta_{\pi}^{l a b}\right)= & \frac{1}{2\left(\left(E_{\pi N}^{q f, l a b}\right)^{2}-\left(q^{l a b}\right)^{2} \cos ^{2} \theta_{\pi}^{l a b}\right)} \\
& \times\left(C_{q f}^{l a b} E_{\pi N}^{q f, l a b} \pm q^{l a b} \cos \theta_{\pi}^{l a b} \sqrt{\left(C_{q f}^{l a b}\right)^{2}-4 m_{\pi}^{2}\left(\left(E_{\pi N}^{q f, l a b}\right)^{2}-\left(q^{l a b}\right)^{2} \cos ^{2} \theta_{\pi}^{l a b}\right)}\right),
\end{aligned}
$$

where we have introduced

$$
C_{q f}^{l a b}=\left(E_{\pi N}^{q f, l a b}\right)^{2}+m_{\pi}^{2}-M^{2}-\left(q^{l a b}\right)^{2}=\left(M_{\pi N}^{q f}\right)^{2}+m_{\pi}^{2}-M^{2},
$$

with $M_{\pi N}^{q f}$ as invariant mass of the active quasi-free $\pi N$-system. In (8) the "plus"-sign should be taken for $0 \leq \theta_{\pi} \leq \pi$, otherwise the "minus"-sign. The corresponding quasi-free missing mass $M_{x}^{q f}$ is given by

$$
M_{x}^{q f}=\sqrt{2 M\left(M_{d}+q_{0}^{l a b}-E_{\pi}^{q f, l a b}\right)} .
$$

In the c.m. system the quasi-free condition for the final spectator momentum reads $p_{s}=\left(\sqrt{M^{2}+q^{2} / 4}, \vec{q} / 2\right)$. The corresponding expressions for the quasi-free pion energy and missing mass are

$$
\begin{aligned}
E_{\pi}^{q f}\left(\theta_{\pi}\right)= & \frac{1}{2\left(\left(E_{\pi N}^{q f}\right)^{2}-q^{2} \cos ^{2} \theta_{\pi}\right)} \\
& \times\left(C_{q f} E_{\pi N}^{q f} \pm q \cos \theta_{\pi} \sqrt{\left(C_{q f}\right)^{2}-4 m_{\pi}^{2}\left(\left(E_{\pi N}^{q f}\right)^{2}-q^{2} \cos ^{2} \theta_{\pi}\right)}\right), \\
M_{x}^{q f}= & \sqrt{2 W\left(W-2 E_{\pi}^{q f}\right)+m_{\pi}^{2}}
\end{aligned}
$$

with

$$
E_{\pi N}^{q f}=W-\sqrt{M^{2}+q^{2} / 4}, \quad \text { and } C_{q f}=\left(E_{\pi N}^{q f}\right)^{2}+m_{\pi}^{2}-M^{2}-q^{2} .
$$

As coordinate system we choose a right-handed orientation with $z$-axis along the photon momentum $\vec{q}$ and $y$-axis perpendicular to the scattering plane along $\vec{k}_{e} \times \vec{k}_{e^{\prime}}$. We distinguish in general three planes: (i) the scattering plane spanned by the incoming and scattered electron momenta, (ii) the pion plane, spanned by the photon and pion momenta, which intersects the scattering plane along the $z$-axis with an angle $\phi_{\pi}$, and (iii) the nucleon plane spanned by the momenta of the two outgoing nucleons intersecting the pion plane along the total momentum of the two nucleons. This is illustrated in Fig. [1]



FIG. 1: Kinematics of single pion electroproduction on the deuteron. 


\section{B. The $T$-matrix}

As in photoproduction, all observables are determined by the $T$-matrix elements of the electromagnetic pion production current $J_{\gamma \pi}$ between the initial deuteron and the final $\pi N N$ states

$$
T_{s m_{s}, \mu m_{d}}=-{ }^{(-)}\left\langle\vec{p}_{1} \vec{p}_{2} s m_{s}, \vec{p}_{\pi}\left|J_{\gamma \pi, \mu}(0)\right| \vec{p}_{d} 1 m_{d}\right\rangle
$$

where $s$ and $m_{s}$ denote the total spin and its projection on the relative momentum $\vec{p}$ of the outgoing two nucleons, and $m_{d}$ correspondingly the deuteron spin projection on the $z$-axis as quantization axis. In the expression on the rhs of (14) non-covariant normalization for the initial deuteron and the final $\pi N N$-states is adopted. As already mentioned, all kinematic quantities related to the $T$-matrix refer to the $\gamma^{*}-d$ c.m. system. Furthermore, the e.m. current is taken in the normalization of the MAID-2003 model. Because of current conservation, one can eliminate either the charge or the longitudinal current component. In this work we have eliminated the longitudinal current component.

Separating the c.m.-motion and making a multipole expansion of the current, its general form is given by

$$
\begin{aligned}
T_{s m_{s} \mu m_{d}}\left(W, Q^{2}, p_{\pi}, \Omega_{\pi}, \Omega_{p}\right) & =-{ }^{(-)}\left\langle\vec{p} s m_{s}, \vec{p}_{\pi}\left|J_{\gamma \pi, \mu}(\vec{q})\right| 1 m_{d}\right\rangle \\
& =\sqrt{2 \pi} \sum_{L} i^{L} \hat{L}^{(-)}\left\langle\vec{p} s m_{s}, \vec{p}_{\pi}\left|\mathcal{O}_{\mu}^{\mu L}\right| 1 m_{d}\right\rangle
\end{aligned}
$$

with $\mu \in\{0, \pm 1\}$ enumerating the spherical current components with the provision that $J_{\gamma \pi, 0}$ is identified with the charge density. Furthermore, we use the notation $\hat{L}=\sqrt{2 L+1}$, and the symbol $\mathcal{O}_{M}^{\mu L}$ comprises charge $\left(C_{M}^{L}\right)$ and transverse multipoles $\left(E_{M}^{L}\right.$ and $\left.M_{M}^{L}\right)$

$$
\mathcal{O}_{M}^{\mu L}=\delta_{\mu 0} C_{M}^{L}+\delta_{|\mu| 1}\left(E_{M}^{L}+\mu M_{M}^{L}\right)
$$

Introducing a partial wave decomposition of the final states, one finds

$$
T_{s m_{s} \mu m_{d}}\left(W, Q^{2}, p_{\pi}, \Omega_{\pi}, \Omega_{p}\right)=e^{i\left(\mu+m_{d}-m_{s}\right) \phi_{\pi}} t_{s m_{s} \mu m_{d}}\left(W, Q^{2}, p_{\pi}, \theta_{\pi}, \theta_{p}, \phi_{p \pi}\right)
$$

where the small $t$-matrix depends besides $W, Q^{2}$ and $p_{\pi}$ only on $\theta_{\pi}, \theta_{p}$, and the relative azimuthal angle $\phi_{p \pi}=\phi_{p}-\phi_{\pi}$. Explicitly one has (for details we refer to [1])

$$
\begin{aligned}
t_{s m_{s} \mu m_{d}}\left(W, Q^{2}, p_{\pi}, \theta_{\pi}, \theta_{p}, \phi_{p \pi}\right)= & \frac{1}{2 \sqrt{2 \pi}} \sum_{L l_{p} j_{p} m_{p} l_{\pi} m_{\pi} J M_{J}} i^{L} \hat{L} \hat{J} \hat{l}_{\pi} \hat{l}_{p} \hat{j}_{p}(-)^{J+l_{p}+j_{p}-s+m_{s}-l_{\pi}} \\
& \times\left(\begin{array}{ccc}
l_{p} & s & j_{p} \\
0 & m_{s} & -m_{s}
\end{array}\right)\left(\begin{array}{ccc}
j_{p} & l_{\pi} & J \\
m_{p} & m_{\pi} & -M_{J}
\end{array}\right)\left(\begin{array}{ccc}
J & L & 1 \\
-M_{J} & \mu & m_{d}
\end{array}\right) \\
& \times\left\langle p p_{\pi}\left(\left(l_{p} s\right) j_{p} l_{\pi}\right) J\left\|\mathcal{O}^{\mu L}\right\| 1\right\rangle d_{m_{s}, m_{p}}^{j_{p}}\left(-\theta_{p}\right) d_{0, m_{\pi}}^{l_{\pi}}\left(-\theta_{\pi}\right) e^{i\left(m_{p}-m_{s}\right) \phi_{p \pi}}
\end{aligned}
$$

We had shown in [1] that, if parity is conserved, the following symmetry relation holds for $\mu= \pm 1$

$$
t_{s-m_{s}-\mu-m_{d}}\left(W, Q^{2}, p_{\pi}, \theta_{\pi}, \theta_{p}, \phi_{p \pi}\right)=(-)^{s+m_{s}+\mu+m_{d}} t_{s m_{s} \mu m_{d}}\left(W, Q^{2}, p_{\pi}, \theta_{\pi}, \theta_{p},-\phi_{p \pi}\right) .
$$

One should note the sign change of $\phi_{p \pi}$ on the right-hand side. It is easy to see that this relation holds also for $\mu=0$, noting that the parity selection rules for charge transitions are the same as for electric ones. As pointed out in [1], all observables can be expressed in terms of the small $t$-matrix elements.

In the present work we include as e.m. current the elementary one-body pion production current of MAID-2003 and consider as FSI the rescattering contributions in the final $N N$ - and $\pi N$-subsystems. Thus as in [2] we split the $T$-matrix into the impulse approximation (IA) $T^{I A}$, where final state interaction effects are neglected, and the rescattering contribution $T^{N N}$ and $T^{\pi N}$ of the two-body $N N$ - and $\pi N$-subsystems, respectively,

$$
T_{s m_{s} \mu m_{d}}=T_{s m_{s} \mu m_{d}}^{I A}+T_{s m_{s} \mu m_{d}}^{N N}+T_{s m_{s} \mu m_{d}}^{\pi N} .
$$

For the IA contribution, where the final state is described by a plane wave, antisymmetrized with respect to the two outgoing nucleons, one has

$$
\begin{aligned}
T_{s m_{s} \mu m_{d}}^{I A} & =\left\langle\vec{p} s m_{s}, \vec{p}_{\pi}\left|\left[j_{\gamma \pi, \mu}(1)+j_{\gamma \pi, \mu}(2)\right]\right| 1 m_{d}\right\rangle \\
& =\sqrt{2} \sum_{m_{s}^{\prime}}\left(\left\langle s m_{s}\left|\left\langle\vec{p}_{1}\left|j_{\gamma \pi, \mu}\left(W_{\gamma N_{1}}, Q^{2}\right)\right| \vec{p}_{d}-\vec{p}_{2}\right\rangle \phi_{m_{s}^{\prime} m_{d}}\left(\frac{1}{2} \vec{p}_{d}-\vec{p}_{2}\right)\right| 1 m_{s}^{\prime}\right\rangle-(1 \leftrightarrow 2)\right)
\end{aligned}
$$


where $j_{\gamma \pi, \mu}$ denotes the elementary pion photoproduction operator of the MAID-2003 model, $W_{\gamma N_{1}}$ the invariant energy of the $\gamma N_{1}$ system, $\vec{p}_{1 / 2}=\left(\vec{q}+\vec{p}_{d}-\vec{p}_{\pi}\right) / 2 \pm \vec{p}$. Furthermore, $\phi_{m_{s} m_{d}}(\vec{p})$ is related to the internal deuteron wave function in momentum space by

$$
\left\langle\vec{p}, 1 m_{s} \mid 1 m_{s}\right\rangle^{(d)}=\phi_{m_{s} m_{d}}(\vec{p})=\sum_{L=0,2} \sum_{m_{L}} i^{L}\left(L m_{L} 1 m_{s} \mid 1 m_{d}\right) u_{L}(p) Y_{L m_{L}}(\hat{p}),
$$

normalized to unity. The two rescattering contributions have a similar structure

$$
\begin{aligned}
T_{s m_{s} \mu m_{d}}^{N N} & =\left\langle\vec{p} s m_{s}, \vec{p}_{\pi}\left|T_{N N} G_{N N}\left[j_{\gamma \pi, \mu}\left(W_{\gamma N_{1}}, Q^{2}\right)+j_{\gamma \pi, \mu}\left(W_{\gamma N_{2}}, Q^{2}\right)\right]\right| 1 m_{d}\right\rangle, \\
T_{s m_{s} \mu m_{d}}^{\pi N} & =\left\langle\vec{p} s m_{s}, \vec{p}_{\pi}\left|T_{\pi N} G_{\pi N}\left[j_{\gamma \pi, \mu}\left(W_{\gamma N_{1}}, Q^{2}\right)+j_{\gamma \pi, \mu}\left(W_{\gamma N_{2}}, Q^{2}\right)\right]\right| 1 m_{d}\right\rangle,
\end{aligned}
$$

where $T_{N N}$ and $T_{\pi N}$ denote respectively the $N N$ and $\pi N$ scattering matrices and $G_{N N}$ and $G_{\pi N}$ the corresponding free two-body propagators.

\section{The differential cross section including polarization observables}

The standard expression of the differential cross section for electroproduction of pions on the deuteron in the one-photon-exchange approximation is

$$
\frac{d^{8} \sigma}{d E_{e^{\prime}} d \Omega_{e^{\prime}} d p_{\pi} d \Omega_{\pi} d \Omega_{p}}=\frac{\alpha_{q e d}}{Q^{4}} \frac{k_{e^{\prime}}}{k_{e}} c\left(W, Q^{2}, p_{\pi}, \Omega_{\pi}, \Omega_{p}\right) \operatorname{tr}\left(T^{\dagger} T \rho_{i}\right),
$$

where $\alpha_{\text {qed }}$ denotes the e.m. fine structure constant, $T$ the reaction matrix, and $\rho_{i}$ the initial state density matrix for the spin degrees of virtual photon and deuteron. The trace refers to all spin degrees of freedom of initial and final states. Furthermore, a kinematic phase space factor is denoted by

$$
c\left(W, Q^{2}, p_{\pi}, \theta_{\pi}, \theta_{p}, \phi_{p \pi}\right)=\frac{M^{2} p^{2} p_{\pi}^{2}}{4(2 \pi)^{4} E_{\pi}\left(E_{12} p+\frac{1}{2} p_{\pi}\left(E_{1}-E_{2}\right) \cos \theta_{p \pi}\right)} .
$$

The density matrix $\rho_{i}$ in (25) is a direct product of the density matrices $\rho^{\gamma^{*}}$ of the virtual photon and $\rho^{d}$ of the deuteron

$$
\rho_{i}=\rho^{\gamma^{*}} \otimes \rho^{d}
$$

One can now proceed in complete analogy on the one hand to deuteron electrodisintegration [13] with respect to the virtual photon and deuteron density matrices and on the other hand to pion photoproduction with respect to the properties of the reaction matrix.

The virtual photon density matrix is determined by the electron kinematics and separates into an unpolarized and a polarized part

$$
\rho_{\lambda \lambda^{\prime}}^{\gamma^{*}}=\rho_{\lambda \lambda^{\prime}}^{0}+h \rho_{\lambda \lambda^{\prime}}^{\prime}
$$

where $|h|$ denotes the degree of longitudinal electron polarization, and $\rho^{0}$ and $\rho^{\prime}$ are given in terms of independent components $\rho_{\alpha}$ and $\rho_{\alpha}^{\prime}(\alpha \in\{L, T, L T, T T\})$ according to the various combinations of longitudinal and transverse polarizations. Its specific form depends on whether one eliminates the charge or the longitudinal current. In the latter case, as used in this work, one has [13]

$$
\begin{aligned}
\rho_{\lambda \lambda^{\prime}}^{0} & =\sum_{\alpha \in\{L, T, L T, T T\}} \delta_{\lambda \lambda^{\prime}}^{\alpha} \rho_{\alpha}, \\
\rho_{\lambda \lambda^{\prime}}^{\prime}= & \sum_{\alpha \in\{L, T, L T, T T\}} \delta_{\lambda \lambda^{\prime}}^{\alpha} \rho_{\alpha}^{\prime},
\end{aligned}
$$

with

$$
\begin{array}{ll}
\delta_{\lambda \lambda^{\prime}}^{L}=\delta_{\lambda \lambda^{\prime}} \delta_{\lambda 0}, & \delta_{\lambda \lambda^{\prime}}^{L T}=\lambda^{\prime} \delta_{\lambda 0}+\lambda \delta_{\lambda^{\prime} 0}, \\
\delta_{\lambda \lambda^{\prime}}^{T}=\delta_{\lambda \lambda^{\prime}}|\lambda|, & \delta_{\lambda \lambda^{\prime}}^{T T}=\delta_{\lambda,-\lambda^{\prime}}|\lambda|, \\
\delta_{\lambda \lambda^{\prime}}^{L}=0, & \delta_{\lambda \lambda^{\prime}}^{L T}=\left|\lambda^{\prime}\right| \delta_{\lambda 0}+|\lambda| \delta_{\lambda^{\prime} 0}, \\
\delta_{\lambda \lambda^{\prime}}^{\prime T}=\delta_{\lambda \lambda^{\prime}} \lambda, & \delta_{\lambda \lambda^{\prime}}^{T T}=0 .
\end{array}
$$


The independent components $\rho_{\alpha}$ and $\rho_{\alpha}^{\prime}$ are given by the well-known expressions [13] (note $Q^{2}=-q_{\nu}^{2}>0$ )

$$
\begin{array}{ll}
\rho_{L}=\rho_{00}^{0}=\beta^{2} Q^{2} \frac{\xi^{2}}{2 \eta}, & \rho_{T}=\rho_{11}^{0}=\frac{1}{2} Q^{2}\left(1+\frac{\xi}{2 \eta}\right), \\
\rho_{L T}=\rho_{01}^{0}=\beta Q^{2} \frac{\xi}{\eta} \sqrt{\frac{\eta+\xi}{8}}, & \rho_{T T}=\rho_{-11}^{0}=-Q^{2} \frac{\xi}{4 \eta}, \\
\rho_{L T}^{\prime}=\rho_{01}^{\prime}=\frac{1}{2} \beta \frac{Q^{2}}{\sqrt{2 \eta}} \xi, \quad \rho_{T}^{\prime}=\rho_{11}^{\prime}=\frac{1}{2} Q^{2} \sqrt{\frac{\eta+\xi}{\eta}},
\end{array}
$$

with

$$
\beta=\frac{q^{\text {lab }}}{q^{c}}, \quad \xi=\frac{Q^{2}}{\left(q^{\text {lab }}\right)^{2}}, \quad \eta=\tan ^{2}\left(\frac{\theta_{e}^{\text {lab }}}{2}\right),
$$

where $\beta$ expresses the boost from the lab system to the frame in which the hadronic current is evaluated and $\vec{q}^{c}$ denotes the momentum transfer in this frame. Here it is the c.m. system, and one has $\vec{q}^{c}=\vec{q}$. As a sideremark, we would like to mention the simple relation to another often used parametrization of the virtual photon density matrix in terms of the quantities $v_{\alpha^{(\prime)}}$ of Ref. [14] (for $\beta=1$ for the lab frame, i.e. $q^{c}=q^{\text {lab }}$ )

$$
\rho_{\alpha}^{(\prime)}=\frac{Q^{2}}{2 \eta} v_{\alpha^{(\prime)}}
$$

where $\alpha \in\{L, T, L T, T T\}$.

Assuming that the deuteron density matrix is diagonal with respect to an orientation axis $\vec{d}$ having spherical angles $\left(\theta_{d}, \phi_{d}\right)$ with respect to the coordinate system associated with the scattering plane in the lab frame, one has with respect to $\vec{d}$ as quantization axis

$$
\rho_{m_{d} m_{d^{\prime}}}^{d}=\frac{1}{\sqrt{3}}(-)^{1-m_{d}} \sum_{I M} \hat{I}\left(\begin{array}{ccc}
1 & 1 & I \\
m_{d}^{\prime} & -m_{d} & M
\end{array}\right) P_{I}^{d} e^{-i M \phi_{d}} d_{M 0}^{I}\left(\theta_{d}\right) .
$$

This means, the deuteron target is characterized by four parameters, namely the vector and tensor polarization parameters $P_{1}^{d}$ and $P_{2}^{d}$, respectively, and by the orientation angles $\theta_{d}$ and $\phi_{d}$. The orientation parameters are related to the probabilities $\left\{p_{m}\right\}$ for finding a deuteron spin projection $m$ on the orientation axis by

$$
P_{I}^{d}=\delta_{I 0}+\sqrt{\frac{3}{2}}\left(p_{1}-p_{-1}\right) \delta_{I 1}+\frac{1}{\sqrt{2}}\left(1-3 p_{0}\right) \delta_{I 2} .
$$

If one chooses the c.m. frame as reference frame as in the present work, one should note that the deuteron density matrix undergoes no change in the transformation from the lab to the c.m. system, since the boost to the c.m. system is collinear with the deuteron quantization axis [12].

Following the same steps as in [1], one finds for the general eight-fold differential cross section for single pion electroproduction with longitudinally polarized electrons

$$
\begin{aligned}
& \frac{d^{8} \sigma}{d E_{e^{\prime}} d \Omega_{e^{\prime}} d p_{\pi} d \Omega_{\pi} d \Omega_{p}}=\frac{\alpha_{q e d}}{Q^{4}} \frac{k_{e^{\prime}}}{k_{e}} \sum_{I=0}^{2} P_{I}^{d}\left[\rho_{L} \sum_{M \geq 0} d_{M 0}^{I}\left(\theta_{d}\right)\left(\tau_{L}^{I M} \cos \left(M \phi_{\pi d}\right)+\sigma_{L}^{I M} \sin \left(M \phi_{\pi d}\right)\right)\right. \\
& \quad+\rho_{T} \sum_{M \geq 0} d_{M 0}^{I}\left(\theta_{d}\right)\left(\tau_{T}^{I M} \cos \left(M \phi_{\pi d}\right)+\sigma_{T}^{I M} \sin \left(M \phi_{\pi d}\right)\right)+\rho_{L T} \sum_{M=-I}^{M} d_{M 0}^{I}\left(\theta_{d}\right)\left(\tau_{L T}^{I M} \cos \phi_{M}+\sigma_{L T}^{I M} \sin \phi_{M}\right) \\
& \quad+\rho_{T T} \sum_{M=-I}^{M} d_{M 0}^{I}\left(\theta_{d}\right)\left(\tau_{T T}^{I M} \cos \psi_{M}+\sigma_{T T}^{I M} \sin \psi_{M}\right)+h \rho_{T}^{\prime} \sum_{M \geq 0} d_{M 0}^{I}\left(\theta_{d}\right)\left(\tau_{T}^{\prime I M} \cos \left(M \phi_{\pi d}\right)+\sigma_{T}^{\prime I M} \sin \left(M \phi_{\pi d}\right)\right) \\
& \left.+h \rho_{L T}^{\prime} \sum_{M=-I}^{M} d_{M 0}^{I}\left(\theta_{d}\right)\left(\tau_{L T}^{\prime I M} \cos \phi_{M}+\sigma_{L T}^{\prime I M} \sin \phi_{M}\right)\right],
\end{aligned}
$$

with

$$
\phi_{\pi d}=\phi_{\pi}-\phi_{d}, \phi_{M}=M \phi_{\pi d}-\phi_{\pi}, \text { and } \psi_{M}=M \phi_{\pi d}-2 \phi_{\pi} .
$$


Again we would like to remind the reader that the electron kinematics refer to the lab system while the final state kinematic variables and the structure functions refer in this work to the c.m. system, which we have chosen for the evaluation of the $T$-matrix. However, we would like to point out that the expression for the differential cross section in (36) holds in general irrespective of which frame of reference collinear with $\vec{q}$ is chosen for the evaluation. Obviously, then the final state variables $p_{\pi}, \theta_{\pi}, \theta_{p}$, and $\phi_{p \pi}$ refer to this frame.

The various exclusive structure functions $\tau_{\alpha}^{(\prime) I M}$ and $\sigma_{\alpha}^{(\prime) I M}$ constitute the polarization observables which determine beam, target and beam-target asymmetries. The structure functions are defined by, not indicating all the kinematic variables $W, Q^{2}, p_{\pi}, \theta_{\pi}, \theta_{p}$, and $\phi_{p \pi}$ on which they depend,

$$
\begin{aligned}
(\tau / \sigma)_{L}^{I M} & = \pm \frac{1}{1+\delta_{M 0}} \Re e / \Im m u_{I M}^{00}, \quad M \geq 0, \\
(\tau / \sigma)_{T}^{I M} & = \pm \frac{1}{1+\delta_{M 0}} \Re e / \Im m\left(u_{I M}^{11}+u_{I M}^{-1-1}\right), \quad M \geq 0 \\
(\tau / \sigma)_{L T}^{I M} & = \pm \Re e / \Im m\left(u_{I M}^{10}-u_{I M}^{0-1}\right), \\
(\tau / \sigma)_{T T}^{I M} & = \pm \Re e / \Im m u_{I M}^{1-1} \\
(\tau / \sigma)_{T}^{I M} & = \pm \frac{1}{1+\delta_{M 0}} \Re e / \Im m\left(u_{I M}^{11}-u_{I M}^{-1-1}\right), \quad M \geq 0, \\
(\tau / \sigma)_{L T}^{I I M} & = \pm \Re e / \Im m\left(u_{I M}^{10}+u_{I M}^{0-1}\right),
\end{aligned}
$$

in terms of the quantities introduced in $[1]$

$$
\begin{aligned}
u_{I M}^{\mu^{\prime} \mu}\left(W, Q^{2}, p_{\pi}, \theta_{\pi}, \theta_{p}, \phi_{p \pi}\right)= & c\left(W, Q^{2}, p_{\pi}, \theta_{\pi}, \theta_{p}, \phi_{p \pi}\right) \frac{\hat{I}}{\sqrt{3}} \sum_{m_{d} m_{d}^{\prime}}(-)^{1-m_{d}}\left(\begin{array}{ccc}
1 & 1 & I \\
m_{d}^{\prime} & -m_{d} & M
\end{array}\right) \\
& \times \sum_{s m_{s}} t_{s m_{s} \mu^{\prime} m_{d}^{\prime}}^{*}\left(W, Q^{2}, p_{\pi}, \theta_{\pi}, \theta_{p}, \phi_{p \pi}\right) t_{s m_{s} \mu m_{d}}\left(W, Q^{2}, p_{\pi}, \theta_{\pi}, \theta_{p}, \phi_{p \pi}\right) .
\end{aligned}
$$

In [1] we have shown that they behave under complex conjugation as

$$
\left(u_{I M}^{\mu^{\prime} \mu}\left(W, Q^{2}, p_{\pi}, \theta_{\pi}, \theta_{p}, \phi_{p \pi}\right)\right)^{*}=(-)^{M} u_{I-M}^{\mu \mu^{\prime}}\left(W, Q^{2}, p_{\pi}, \theta_{\pi}, \theta_{p}, \phi_{p \pi}\right) .
$$

From this property follows in particular that the $u_{I 0}^{\mu \mu}$ are real. Furthermore, the $u_{I M}^{\mu^{\prime} \mu}$ possess the symmetry property

$$
u_{I M}^{-\mu^{\prime}-\mu}\left(W, Q^{2}, p_{\pi}, \theta_{\pi}, \theta_{p}, \phi_{p \pi}\right)=(-)^{I+M+\mu^{\prime}+\mu} u_{I-M}^{\mu^{\prime} \mu}\left(W, Q^{2}, p_{\pi}, \theta_{\pi}, \theta_{p},-\phi_{p \pi}\right),
$$

which yields in combination with (45)

$$
u_{I M}^{-\mu^{\prime}-\mu}\left(W, Q^{2}, p_{\pi}, \theta_{\pi}, \theta_{p}, \phi_{p \pi}\right)=(-)^{I+\mu^{\prime}+\mu}\left(u_{I M}^{\mu \mu^{\prime}}\left(W, Q^{2}, p_{\pi}, \theta_{\pi}, \theta_{p},-\phi_{p \pi}\right)\right)^{*} .
$$

At the photon point, one finds the following equivalencies to the corresponding quantities in pion photoproduction defined in [1]

$$
(\tau / \sigma)_{T}^{I M}=\frac{q_{0} W}{\pi^{2} E_{d}}(\tau / \sigma)_{I M}^{0}, \quad(\tau / \sigma)_{T T}^{I M}=\frac{q_{0} W}{\pi^{2} E_{d}}(\tau / \sigma)_{I M}^{l}, \quad(\tau / \sigma)_{T}^{I I M}=\frac{q_{0} W}{\pi^{2} E_{d}}(\tau / \sigma)_{I M}^{c},
$$

where obviously the kinematic variables on the right hand sides should refer to the same reference frame then on the left hand sides.

For the semi-exclusive reaction $\vec{d}\left(\vec{e}, e^{\prime} \pi\right) N N$, where besides the scattered electron only the produced pion is detected, the basic quantities are obtained from the $u_{I M}^{\mu^{\prime} \mu}\left(p_{\pi}, \theta_{\pi}, \theta_{p}, \phi_{p \pi}\right)$ in (44) by integration over $d \Omega_{p}$. Thus we introduce

$$
\begin{aligned}
U_{I M}^{\mu^{\prime} \mu}\left(W, Q^{2}, p_{\pi}, \theta_{\pi}\right)= & \int d \Omega_{p} u_{I M}^{\mu^{\prime} \mu}\left(W, Q^{2}, p_{\pi}, \theta_{\pi}, \theta_{p}, \phi_{p \pi}\right) \\
= & \frac{\hat{I}}{\sqrt{3}} \int d \Omega_{p} c\left(W, Q^{2}, p_{\pi}, \theta_{\pi}, \theta_{p}, \phi_{p \pi}\right) \sum_{m_{d} m_{d}^{\prime}}(-)^{1-m_{d}}\left(\begin{array}{ccc}
1 & 1 & I \\
m_{d}^{\prime} & -m_{d} & M
\end{array}\right) \\
& \times \sum_{s m_{s}}\left(t_{s m_{s} \mu^{\prime} m_{d}^{\prime}}\left(W, Q^{2}, p_{\pi}, \theta_{\pi}, \theta_{p}, \phi_{p \pi}\right)\right)^{*} t_{s m_{s} \mu m_{d}}\left(W, Q^{2}, p_{\pi}, \theta_{\pi}, \theta_{p}, \phi_{p \pi}\right) .
\end{aligned}
$$


From the properties of (45) and (46) one obtains corresponding properties

$$
\left(U_{I M}^{\mu^{\prime} \mu}\right)^{*}=(-)^{M} U_{I-M}^{\mu \mu^{\prime}} \quad \text { and } \quad U_{I M}^{-\mu^{\prime}-\mu}=(-)^{I+M+\mu+\mu^{\prime}}\left(U_{I M}^{\mu \mu^{\prime}}\right)^{*} .
$$

Combining them leads to

$$
U_{I M}^{-\mu^{\prime}-\mu}=(-)^{I+\mu+\mu^{\prime}}\left(U_{I M}^{\mu \mu^{\prime}}\right)^{*}
$$

An important consequence of this latter property is that, according to (38) through (43), the following integrated structure functions vanish for $\alpha \in\{L, T, L T, T T\}$

$$
\begin{aligned}
& \int d \Omega_{p} \tau_{\alpha}^{1 M}=0 \text { and } \int d \Omega_{p} \sigma_{\alpha}^{\prime M}=0 \\
& \int d \Omega_{p} \sigma_{\alpha}^{I M}=0 \text { and } \int d \Omega_{p} \tau_{\alpha}^{\prime I M}=0 \text { for } I=0,2 .
\end{aligned}
$$

The remaining semi-exclusive structure functions govern the six-fold semi-exclusive differential cross section for which we find as final form

$$
\begin{aligned}
\frac{d^{6} \sigma}{d E_{e^{\prime}} d \Omega_{e^{\prime}} d p_{\pi} d \Omega_{\pi}}= & \frac{\alpha_{q e d}}{Q^{4}} \frac{k_{e^{\prime}}}{k_{e}} \sum_{I=0}^{2} P_{I}^{d}\left[\rho_{L} \sum_{M \geq 0} d_{M 0}^{I}\left(\theta_{d}\right) \widetilde{f}_{L}^{I M} \cos \left(M \phi_{\pi d}-\delta_{I 1} \frac{\pi}{2}\right)\right. \\
& +\rho_{T} \sum_{M \geq 0} d_{M 0}^{I}\left(\theta_{d}\right) \widetilde{f}_{T}^{I M} \cos \left(M \phi_{\pi d}-\delta_{I 1} \frac{\pi}{2}\right)+\rho_{L T} \sum_{M=-I}^{M} d_{M 0}^{I}\left(\theta_{d}\right) \widetilde{f}_{L T}^{I M} \cos \left(\phi_{M}-\delta_{I 1} \frac{\pi}{2}\right) \\
& +\rho_{T T} \sum_{M=-I}^{M} d_{M 0}^{I}\left(\theta_{d}\right) \tilde{f}_{T T}^{I M} \cos \left(\psi_{M}-\delta_{I 1} \frac{\pi}{2}\right)+h \rho_{T}^{\prime} \sum_{M \geq 0} d_{M 0}^{I}\left(\theta_{d}\right) \widetilde{f}_{T}^{\prime I M} \sin \left(M \phi_{\pi d}+\delta_{I 1} \frac{\pi}{2}\right) \\
& \left.+h \rho_{L T}^{\prime} \sum_{M=-I}^{M} d_{M 0}^{I}\left(\theta_{d}\right) \widetilde{f}_{L T}^{I M} \sin \left(\phi_{M}+\delta_{I 1} \frac{\pi}{2}\right)\right],
\end{aligned}
$$

where the angles $\phi_{\pi d}, \phi_{M}$, and $\psi_{M}$ are defined in (37). The semi-exclusive structure functions $\tilde{f}_{\alpha}^{(\prime) I M}$ are given by

$$
\begin{array}{ll}
\widetilde{f}_{L}^{I M}=\frac{i^{\delta_{I 1}}}{1+\delta_{M 0}} U_{I M}^{00}, & \tilde{f}_{T}^{I M}=\frac{2}{1+\delta_{M 0}} \Re e\left(i^{\delta_{I 1}} U_{I M}^{11}\right), \\
\tilde{f}_{L T}^{I M}=2 \Re e\left(i^{\delta_{I 1}} U_{I M}^{10}\right), & \tilde{f}_{T T}^{I M}=\Re e\left(i^{\delta_{I 1}} U_{I M}^{1-1}\right), \\
\tilde{f}_{L T}^{\prime I M}=2 \Im m\left(i^{\delta_{I 1}} U_{I M}^{10}\right), & \tilde{f}_{T}^{\prime I M}=\frac{2}{1+\delta_{M 0}} \Im m\left(i^{\delta_{I 1}} U_{I M}^{11}\right) .
\end{array}
$$

They depend on $W, Q^{2}, p_{\pi}$, and $\theta_{\pi}$. Because $U_{I 0}^{11}$ is real according to (50), the structure functions $\tilde{f}_{T}^{10}$ and $\tilde{f}_{T}^{\prime 20}$ vanish identically. We would like to point out that for forward and backward pion emission, i.e. for $\theta_{\pi}=0$ and $\pi$, the following structure functions have to vanish

$$
\widetilde{f}_{L}^{I M}=0 \text { and } \widetilde{f}_{T}^{(\prime) I M}=0 \text { for } M \neq 0, \widetilde{f}_{L T}^{(\prime) I M}=0 \text { for } M \neq 1, \text { and } T_{T T}^{I M}=0 \text { for } M \neq 2,
$$

because in that case the differential cross section cannot depend on $\phi_{\pi}$, since at $\theta_{\pi}=0$ or $\pi$ the azimuthal angle $\phi_{\pi}$ is undefined or arbitrary. This feature can also be shown by straightforward evaluation of $U_{I M}^{\mu^{\prime} \mu}$ using the explicit representation of the $t$-matrix in (18) as shown in [1].

In case that only the direction of the outgoing pion is measured and not its momentum, the corresponding differential cross section $d^{5} \sigma /\left(d E_{e^{\prime}} d \Omega_{e^{\prime}} d \Omega_{\pi}\right)$ is given by an expression formally analogous to (54) where only the above structure functions are integrated over the pion momentum, i.e., by the replacement

$$
\widetilde{f}_{\alpha}^{(\prime) I M}\left(W, Q^{2}, p_{\pi}, \theta_{\pi}\right) \rightarrow f_{\alpha}^{(\prime) I M}\left(W, Q^{2}, \theta_{\pi}\right)=\int_{0}^{p_{\pi, \max }} d p_{\pi} \widetilde{f}_{\alpha}^{(\prime) I M}\left(p_{\pi}, \theta_{\pi}\right)
$$

for $\alpha \in\{L, T, L T, T T\}$. The upper integration limit is listed in (6). 
The general totally inclusive cross section with respect to the hdronic final state $d\left(e, e^{\prime}\right) \pi N N$ is obtained from (54) by integration over both $p_{\pi}$ and $\Omega_{\pi}$ resulting in

$$
\begin{aligned}
\frac{d^{3} \sigma}{d E_{e^{\prime}} d \Omega_{e^{\prime}}}= & \frac{\alpha_{q e d}}{Q^{4}} \frac{k_{e^{\prime}}}{k_{e}}\left[\rho_{L} F_{L}^{00}+\rho_{T} F_{T}^{00}+P_{1}^{d}\left(h \rho_{T}^{\prime} F_{T}^{\prime 10} d_{00}^{1}\left(\theta_{d}\right)+\left[\rho_{L T} F_{L T}^{11}+h \rho_{L T}^{\prime} F_{L T}^{\prime 11}\right] d_{10}^{1}\left(\theta_{d}\right)\right) \cos \phi_{d}\right. \\
& \left.+P_{2}^{d}\left(\left[\rho_{L} F_{L}^{20}+\rho_{T} F_{T}^{20}\right] d_{00}^{2}\left(\theta_{d}\right)+\rho_{L T} F_{L T}^{21} d_{10}^{2}\left(\theta_{d}\right) \cos \phi_{d}+\rho_{T T} F_{T T}^{22} d_{20}^{2}\left(\theta_{d}\right) \cos \left(2 \phi_{d}\right)\right)\right]
\end{aligned}
$$

in terms of various form factors

$$
\begin{aligned}
F_{\alpha}^{(\prime) I M}\left(W, Q^{2}\right) & =\int d \Omega_{\pi} \int_{0}^{p_{\pi, \max }} d p_{\pi} \widetilde{f}_{\alpha}^{(\prime) I M}\left(W, Q^{2}, p_{\pi}, \theta_{\pi}\right) \\
& =2 \pi \int d\left(\cos \theta_{\pi}\right) f_{\alpha}^{(\prime) I M}\left(W, Q^{2}, \theta_{\pi}\right) .
\end{aligned}
$$

At the photon point one obtains from (48) the following relations to the contributions to the total photoproduction cross section as listed in eq. (83) of [1]

$$
F_{T}^{00}=\frac{q_{0} W}{\pi^{2} E_{d}} \sigma_{0}, \quad F_{T}^{20}=\frac{q_{0} W}{\pi^{2} E_{d}} \bar{T}_{20}^{0}, \quad F_{T}^{\prime 10}=\frac{q_{0} W}{\pi^{2} E_{d}} \bar{T}_{10}^{c}, \quad F_{T T}^{22}=\frac{q_{0} W}{\pi^{2} E_{d}} \bar{T}_{22}^{l} .
$$

Finally, we would like to point out that for coherent electroproduction of $\pi^{0}$ on the deuteron formally the same expression as in (54) holds with structure functions, which are defined in analogy to (55) with the replacement

$$
U_{I M}^{\mu^{\prime} \mu} \rightarrow c\left(W, Q^{2}, \theta_{\pi}\right) \frac{\hat{I}}{\sqrt{3}} \sum_{m_{d} m_{d}^{\prime}}(-)^{1-m_{d}}\left(\begin{array}{ccc}
1 & 1 & I \\
m_{d}^{\prime} & -m_{d} & M
\end{array}\right) \sum_{m_{d}^{\prime \prime}} t_{m_{d}^{\prime \prime} \mu^{\prime} m_{d}^{\prime}}^{*}\left(W, Q^{2}, \theta_{\pi}\right) t_{m_{d}^{\prime \prime} \mu m_{d}}\left(W, Q^{2}, \theta_{\pi}\right),
$$

where $c\left(W, Q^{2}, \theta_{\pi}\right)$ denotes a kinematic factor.

\section{RESULTS AND DISCUSSION}

As elementary pion production amplitude we use the MAID-2003 model which is parametrized in terms of invariant amplitudes allowing the evaluation in any frame of reference. As in photoproduction, one encounters the principal problem of off-shell continuation. In the present work this problem is neglected by assuming on-shell kinematics for the struck nucleon and the pion in the final state, because the MAID-amplitudes do not allow an off-shell extrapolation. For the evaluation of the MAID amplitudes the invariant $\pi N$ energy $W_{\pi N}$, the squared four momentum transfer $Q^{2}$, and the pion angle $\theta_{\pi N}$ in the $\pi N$ c.m. system have to be specified. While $Q^{2}$ is given by the virtual photon, one has to determine $W_{\pi N}$ and $\theta_{\pi N}$ from the kinematics of the active nucleon in the $\gamma^{*}-d$ c.m. system. For this purpose we assume as just mentioned that the four momenta $p_{\pi}$ and $p_{f}$ of pion and active nucleon, respectively, in the final state obey the on-shell condition. Then the needed $\pi N \mathrm{c} . \mathrm{m}$. variables are obtained by a Lorentz transformation with with boost parameter $\vec{\beta}=\left(\vec{p}_{\pi}+\vec{p}_{f}\right) /\left(E_{\pi}+E_{f}\right)$. The energy and momentum of the initial off-shell nucleon then are determined through the energy-momentum conservation at the elementary vertex, i.e. $p_{i}=p_{\pi}+p_{f}-q$.

The explicit calculation of the $N N$-rescattering contribution follows the same approach as in photoproduction [2] by using the separable representation of the realistic Paris potential from [15] and include all partial waves up to ${ }^{3} D_{3}$. From previous results on photoproduction it is expected that any realistic $N N$-potential model will give very similar results. Thus the use of the Paris potential is not crucial. Also with respect to the question whether the use of a nonrelativistic $N N$-potential can be justified in view of the high energies involved, we can refer to the remark in [2]. Similarly, we use for the evaluation of $\pi N$-rescattering a realistic separable representation of the $\pi N$ interaction from [16] and take into account all partial waves up to $l=2$.

\section{A. Survey on semi-exclusive structure functions}

We will start with a general survey on the properties of the semi-exclusive structure functions, unpolarized as well as polarized. With respect to the two possible charged pion channels we will consider here $\pi^{+}$production only, because the role of hadronic FSI in $\pi^{-}$production is expected to be very similar to the one in $\pi^{+}$production according to the results in photoproduction [2]. 

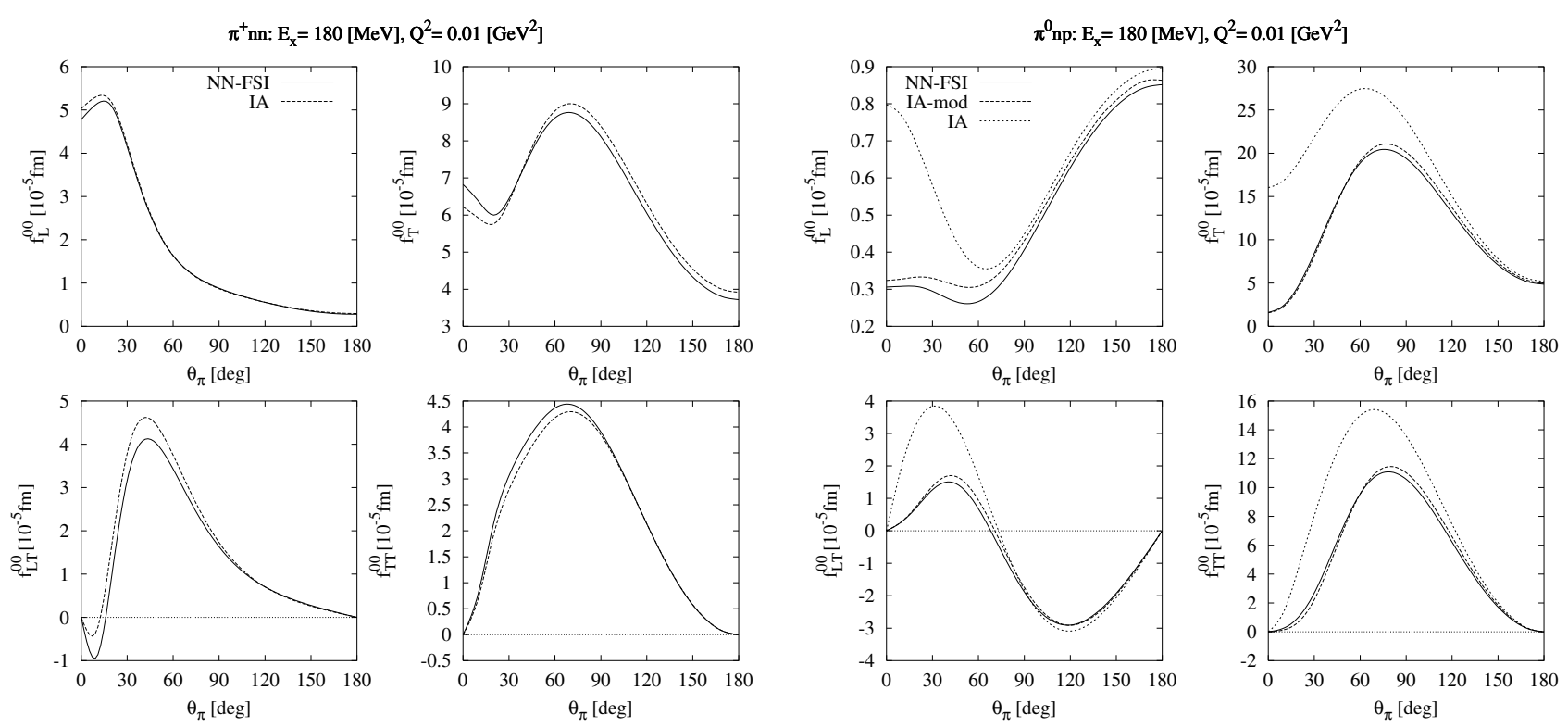

FIG. 2: Unpolarized structure functions for $\pi^{+}$(left four panels) and $\pi^{0}$ electroproduction (right four panels) at excitation energy $E_{x}=180 \mathrm{MeV}$ and squared four momentum transfer $Q^{2}=0.01 \mathrm{GeV}^{2}$ with $N N$-rescattering in the final state (NN-FSI) and without (IA). For $\pi^{0}$ production results for the modified IA are also given (IA-mod).

\section{Unpolarized semi-exclusive structure functions}

All four unpolarized structure functions for $\pi^{+}$and $\pi^{0}$ electroproduction are shown in Fig.2 in IA and with inclusion of only $N N$-rescattering for an excitation energy $E_{x}=180 \mathrm{MeV}\left(E_{x}=W-2 M-m_{\pi}\right)$, which is in the region of the $\Delta$-resonance, and for a quite low squared four-momentum transfer $Q^{2}=0.01 \mathrm{GeV}^{2}$. The dependence on $Q^{2}$ will be discussed later. The reason that we show only the influence of $N N$-rescattering is that $\pi N$-rescattering is very small. This fact has been noticed already by many authors [2, 18, 19, 20] for the case of incoherent photoproduction of pions on the deuteron. Close to the threshold it follows from the fact that the characteristic scale for $\pi N$-FSI effects is given by the small ratio of the pion-nucleon scattering length to the deuteron radius, i.e. by $a_{\pi N} / R_{d} \ll 1$. At higher energies the insignificance of pion rescattering is related to the smallness of the parameter $\left(p R_{d}\right)^{-1}$, where $p$ is a characteristic momentum of the rescattered pion. As a consequence, the $\pi N$-interaction is much less effective in comparison to $N N$-rescattering.

One readily notes in Fig. 2 that for charged pion production the rescattering effects are in general quite small. It has already been mentioned that they arise predominantly from $N N$-rescattering while $\pi N$-rescattering is almost negligible. The only exception is the near threshold region as will be shown below. For $\pi^{+}$production $f_{L}^{00}$ exhibits a distinct forward peak while $f_{T}^{00}$ possesses a much broader angular distribution with a maximum around $75^{\circ}$. In $f_{L}^{00}$ significant FSI effects appear only at small angles below $30^{\circ}$ where they result in a small decrease. In contrast to this, one notes in $f_{T}^{00}$ FSI effects over the whole angular range, in forward direction a slight decrease and above $50^{\circ}$ a small increase. The interference structure functions are of the same magnitude than the diagonal ones. Both exhibit a maximum, around $40^{\circ}$ for $f_{L T}^{00}$ with a smaller widths and near $70^{\circ}$ for $f_{T T}^{00}$ with a broader distribution. FSI results in a slight reduction in $f_{L T}^{00}$ and a small enhancement in $f_{T T}^{00}$.

The unpolarized structure function for neutral pion production in Fig. 2 exhibit quite a different behavior. The influence of FSI is dramatic which, however, stems predominantly from the well-known fact, that in IA a large fraction of coherent production is included because the final $N N$-plane wave is not orthogonal to the deuteron bound state wave function. As is discussed in detail in [2] the effect of this non-orthogonality can be eliminated by applying a modified IA where the deuteron wave function component is projected out from the final $N N$-plane wave (see Appendix B of [2]). The additional influence of FSI then is indeed quite small and comparable to charged pion production. In contrast to $\pi^{+}$production, $f_{L}^{00}$ exhibits a pronounced peak in backward direction. However, in absolute size this structure function is much smaller than $f_{T}^{00}$ and thus it is not surprising that FSI is noteable over the whole angular region, particularly sizeable near the minimum around $50^{\circ}$. On the other hand, $f_{T}^{00}$ and the interference structure functions $f_{L T}^{00}$ and $f_{T T}^{00}$ as well show very little FSI effects. Interesting is the pronounced forward-backward asymmetry of $f_{L T}^{00}$.

As already mentioned, the $\pi N$-final state interaction plays a role only near threshold. This is demonstrated in Fig. 3 

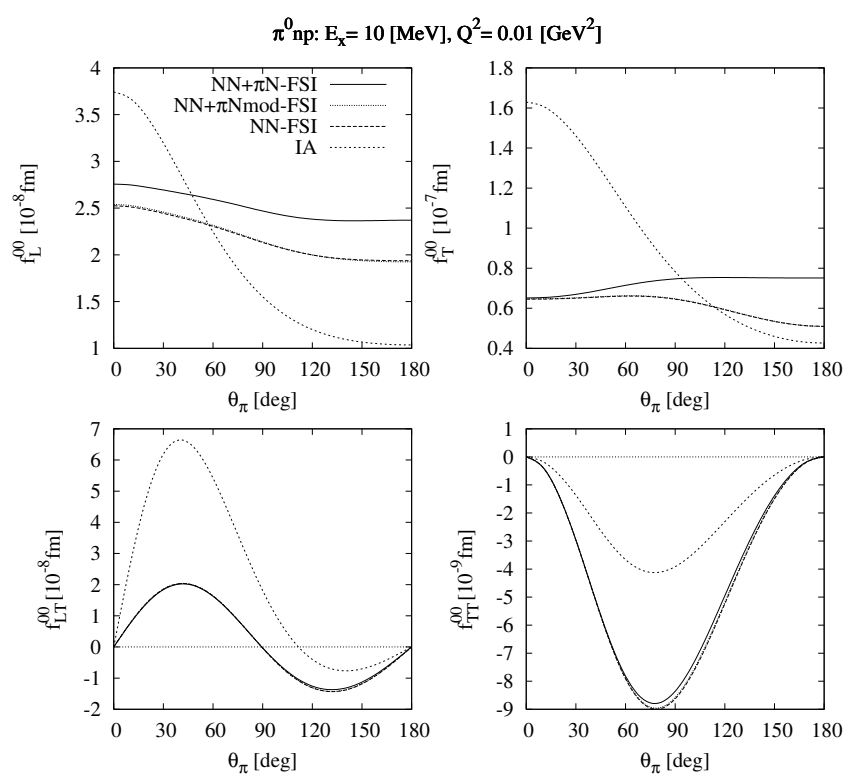


imation (IA) on unpolarized structure functions of $\pi^{0}$ electropoduction near threshold at excitation energy $E_{x}=10 \mathrm{MeV}$ and squared four momentum transfer $Q^{2}=0.01 \mathrm{GeV}^{2}$. For the curve labeled "NN-FSI $+\pi$ Nmod" the charge exchange contribution to the $\pi N$ FSI has been switched off.

where we show for $\pi^{0}$ production the unpolarized structure functions in the near threshold region, i.e. $10 \mathrm{MeV}$ above threshold. In particular, the diagonal structure functions $f_{L}^{00}$ and $f_{T}^{00}$ show quite a significant enhancement from $\pi N$ rescattering. In contrast to this, the interference structure functions are very little affected by the additional $\pi N$-FSI. But as soon as the excitation energy approaches the first resonance region, the influence of $\pi N$-FSI dies out rapidly. The sizeable influence of $\pi N$-FSI in $\pi^{0}$ production shown in Fig. [2 is mostly due to the strong suppression of the IA
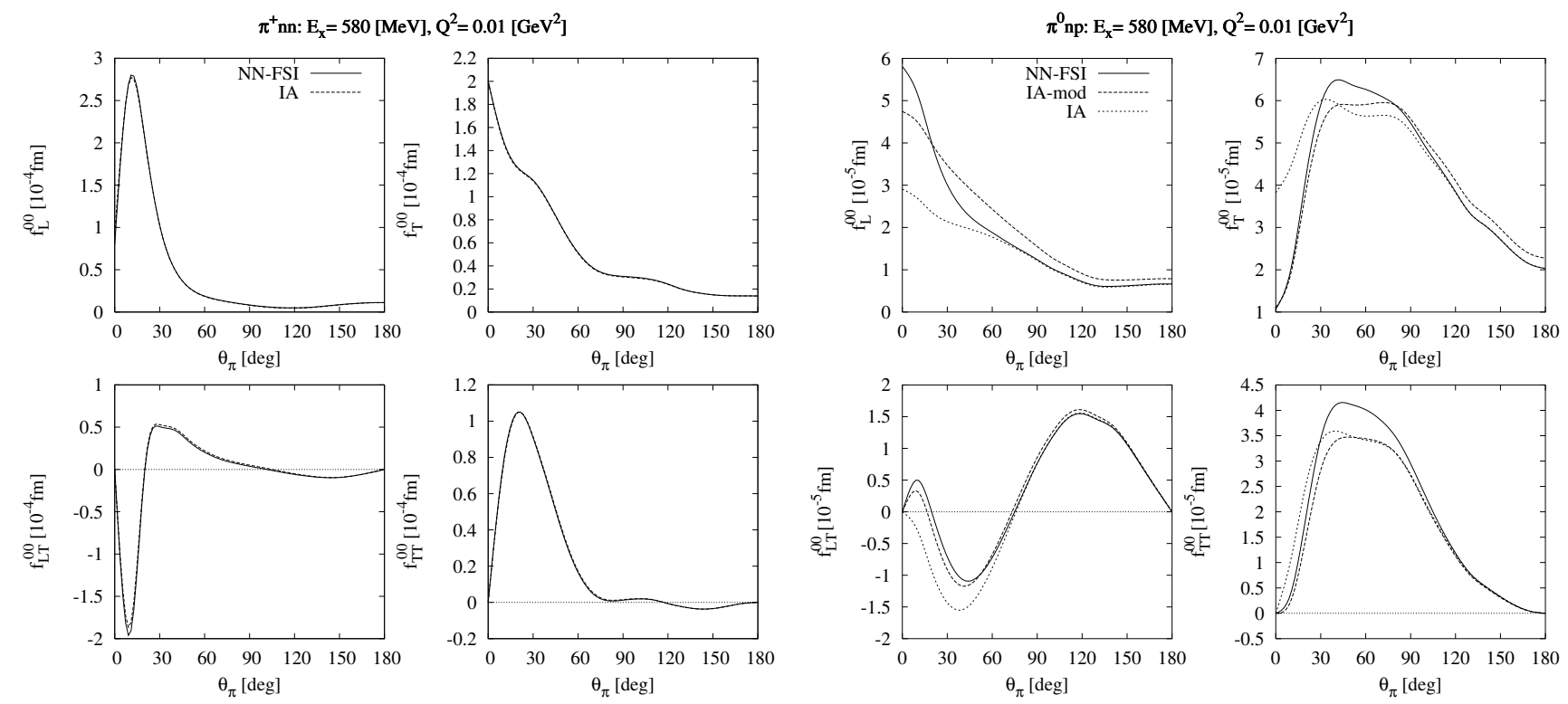

FIG. 4: Unpolarized structure functions for $\pi^{+}$(left four panels) and $\pi^{0}$ electroproduction (right four panels) at excitation energy $E_{x}=580 \mathrm{MeV}$ and squared four momentum transfer $Q^{2}=0.01 \mathrm{GeV}^{2}$ with $N N$-rescattering in the final state (NN-FSI) and without (IA). For $\pi^{0}$ production results for the modified IA are also given.

cross section in the $\pi^{0}$ channel in the near threshold region. As is well known, at very low kinetic energies of the active $\pi N$ system the dipole amplitude $E_{0+}$ in the neutral channel is about an order of magnitude smaller than the one for 
$\pi^{ \pm}$production. As a result, the dominant $\pi N$ rescattering effect in $\pi^{0}$ production is the charge-exchange mechanism, in which the production of a charged pion on one of the nucleons is followed by a charge-exchange rescattering on the second nucleon. This fact is demonstrated in Fig. 2 by the curve labeled "NN-FSI $+\pi$ Nmod" representing the calculation for which this charge-exchange mechanism has been switched off.

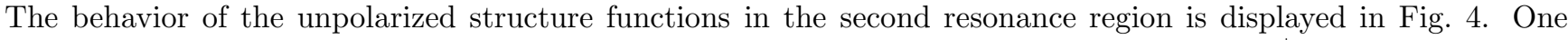
readily notes a significant change in the angular distributions compared to the $\Delta$-regions. For $\pi^{+}$production, a more pronounced forward peaking is seen, particularly for $f_{T}^{00}$ and $f_{T T}^{00}$. Final state interaction effects are almost negligible. This is not the case for $\pi^{0}$ production, where FSI still has a significant influence, although much less than in the $\Delta$-region. Also here a large fraction of FSI effects is eliminated by the modified IA. The angular distributions are still broad except for $f_{L}^{00}$ where one notes a forward increase.
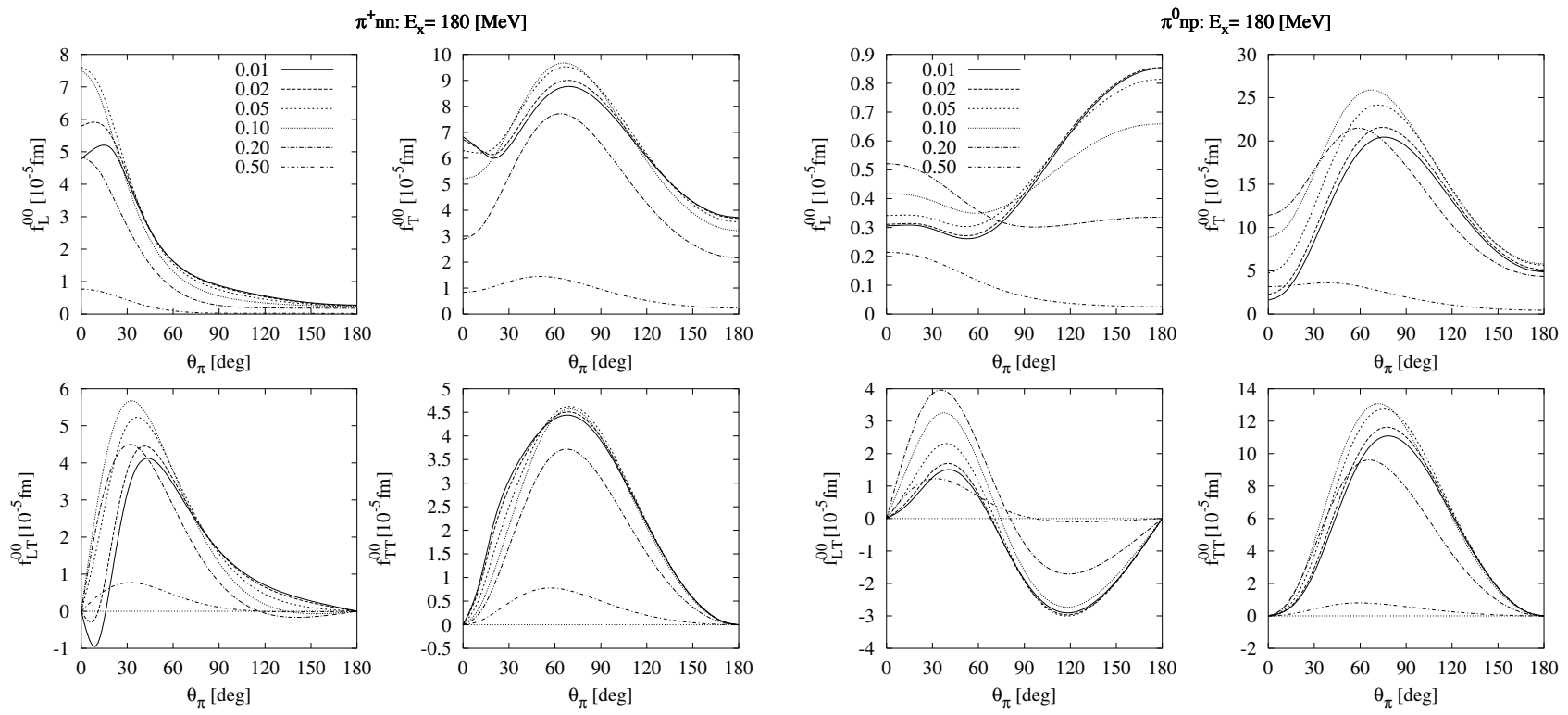

FIG. 5: $Q^{2}$-dependence of unpolarized structure functions for $\pi^{+}$(left four panels) and $\pi^{0}$ electroproduction (right four panels) at excitation energy $E_{x}=180 \mathrm{MeV}$ and various squared four momentum transfers $Q^{2}=0.01,0.02,0.05,0.1,0.2$, and $0.5 \mathrm{GeV}^{2}$ as indicated in the legend with inclusion of $N N$-rescattering in the final state.

The dependence on $Q^{2}$ is shown in Fig. [5] for $E_{x}=180 \mathrm{MeV}$ on the $\Delta$-resonance and in Fig. [6 for $E_{x}=580 \mathrm{MeV}$ in the second resonance region. At both energies, the angular behavior of the structure functions for different values of $Q^{2}$ look quite similar. For $\pi^{+}$production the shape of the four structure functions remains unchanged qualitatively except for $f_{T T}^{00}$ at the lowest $Q^{2}$-value, only the size varies with $Q^{2}$. In $f_{L}^{00}$ one first notes a slight increase going from $Q^{2}=0.01 \mathrm{GeV}^{2}$ to $Q^{2}=0.05 \mathrm{GeV}^{2}$ but at higher $Q^{2}$ a steady rapid decrease. The transverse structure function shows a general decrease in size, whereas for $f_{L T}^{00}$ the decrease starts only after $Q^{2}=0.05 \mathrm{GeV}^{2}$. The transverse-transverse interference function first increases slightly, then remains almost constant up to $Q^{2}=0.1 \mathrm{GeV}^{2}$ and finally becomes rapidly smaller at higher $Q^{2}$.

In contrast to this, the structure functions for neutral pion production in the right panels of Fig. 5 show quite a different $Q^{2}$-dependence. Here, $f_{L}^{00}$ exhibits a strong increase at forward angles with a corresponding decrease in backward direction. For $f_{T}^{00}$ the maximum is slightly shifted towards smaller angles while the amplitude remains constant up to $Q^{2}=0.07 \mathrm{GeV}^{2}$ and then falls off rapidly at higher $Q^{2}$. The $L T$-interference function shows an increase of the forward maximum and a decrease of the backward minimum. Only for $f_{T T}^{00}$ the shape remains unchanged while the amplitude falls off with increasing $Q^{2}$. Qualitatively, one finds a similar behavior at the higher excitation energy $E_{x}=580 \mathrm{MeV}$ in the second resonance region (Fig. 6).

\section{Polarized semi-exclusive structure functions for beam and target polarization}

For a longitudinally polarized electron beam and a polarized deuteron target the number of structure functions increases significantly. For that reason we show them in separate figures for longitudinal, transverse and the interference ones. The polarized longitudinal structure functions in Fig. 7 for $\pi^{+}$production exhibit in general a pronounced forward peaking, except for $f_{L}^{11}$ showing an oscillatory behavior with a maximum near $60^{\circ}$. Since $f_{L}^{20}$ and $f_{L}^{21}$ are 


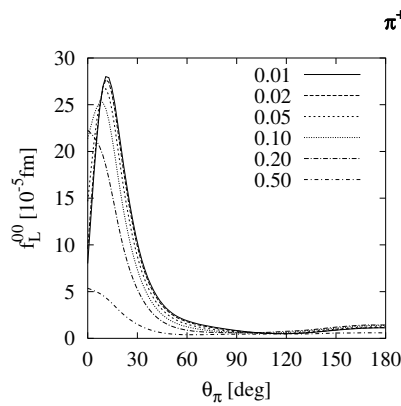

$\pi^{+} \mathrm{nn}: \mathrm{E}_{\mathrm{x}}=580[\mathrm{MeV}]$
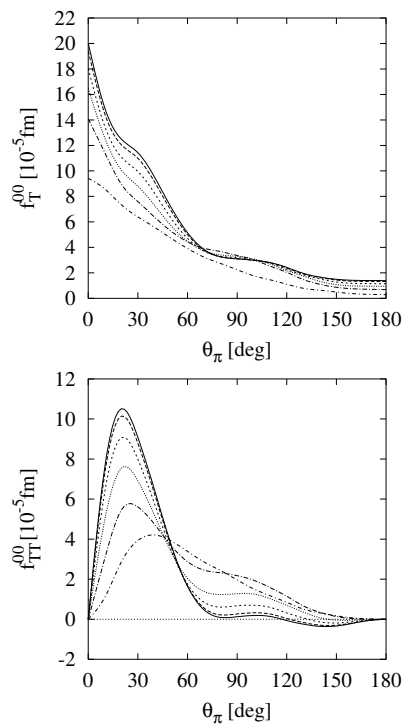

$\pi^{0}{ }^{0} \mathrm{np} \mathrm{E}_{\mathrm{x}}=580[\mathrm{MeV}]$
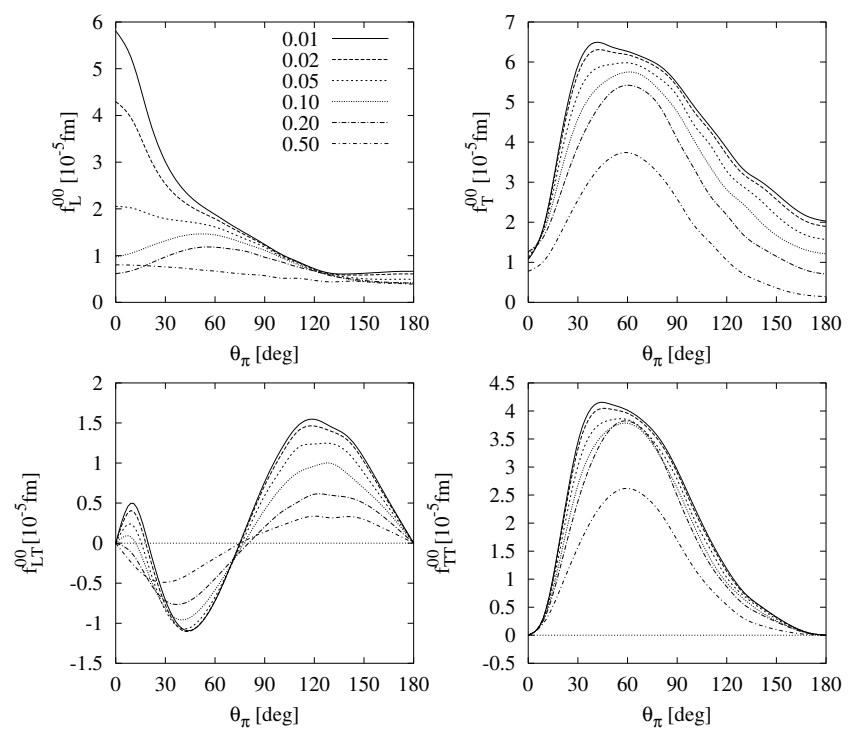

FIG. 6: As Fig. [0 at excitation energy $E_{x}=580 \mathrm{MeV}$.
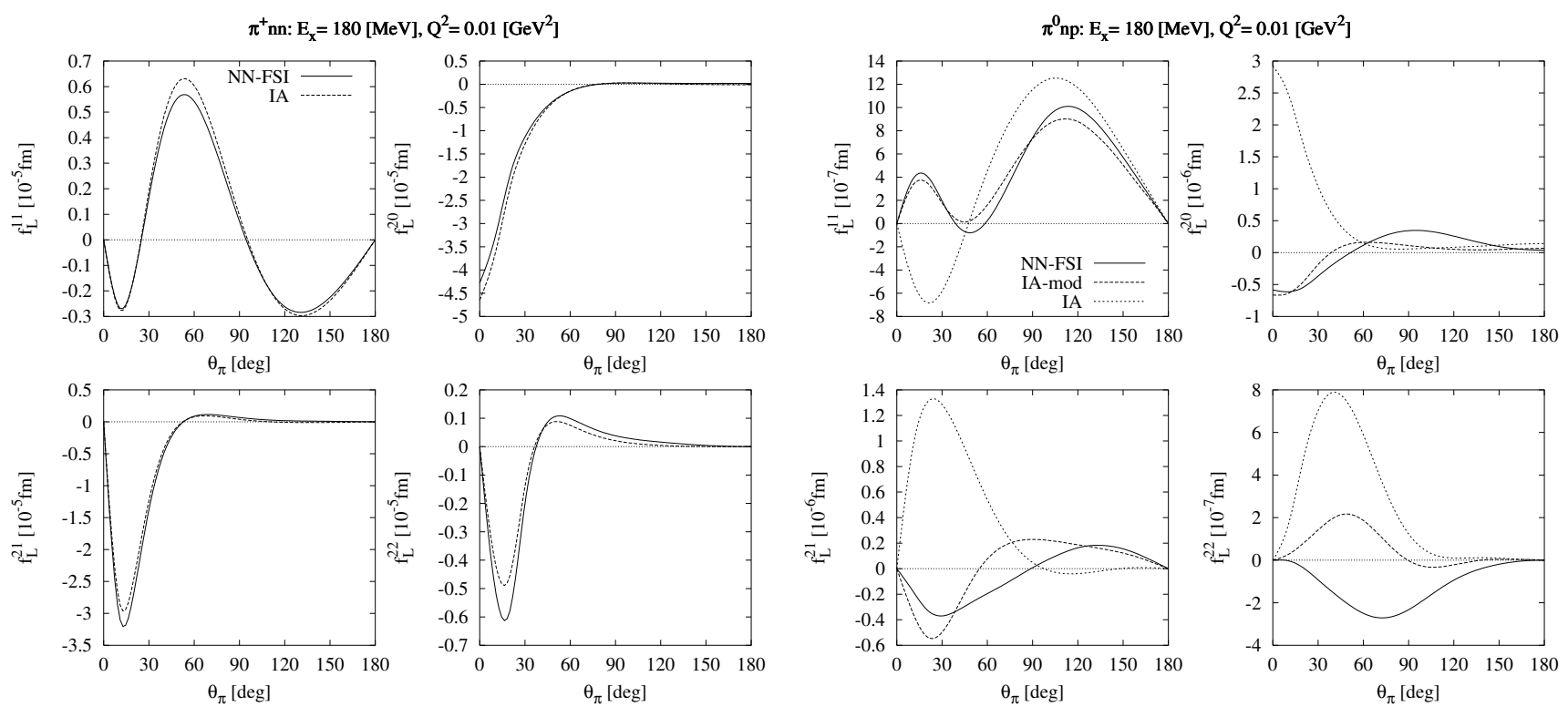

FIG. 7: Polarized longitudinal structure functions for $\pi^{+}$(left four panels) and $\pi^{0}$ electroproduction (right four panels) at excitation energy $E_{x}=180 \mathrm{MeV}$ and squared four momentum transfer $Q^{2}=0.01 \mathrm{GeV}^{2}$ with $N N$-rescattering in the final state (NN-FSI) and without (IA). For $\pi^{0}$ production results for the modified IA are also given.

comparable in size to the unpolarized $f_{L}^{00}$, one expects a sizeable dependence on the tesor polarization of an oriented deuteron target. This has been pointed out already in [7]. The other two structure functions, $f_{L}^{11}$ and $f_{L}^{22}$, are smaller by about a factor 5 . For neutral pion production the polarized structure functions are an order of magnitude smaller. They exhibit a much broader angular distribution. FSI effects are very strong as in the unpolarized case which, however, are again largely reduced by using the modified IA. But the remaining FSI effects are still quite significant, in particular for $f_{L}^{21}$ and $f_{L}^{22}$.

Much broader angular distributions show many of the transverse structure functions in Fig. 8 For $\pi^{+}$production only $f_{T}^{20}$ and $f_{T}^{22}$ exhibit a strong forward peaking. Comparable in size to the unpolarized $f_{T}^{00}$ is $f_{T}^{11}$, and also $f_{T}^{20}$ is sizeable at small angles. Remarkable is the fact that $f_{T}^{11}$ is almost independent from FSI, whereas $f_{T}^{21}$ is quite sensitive to FSI. Compared to $\pi^{+}$one finds a different, more oscillatory angular dependence. The largest one is $f_{T}^{11}$ while the other three structure functions are considerably smaller. Additional FSI effects beyond the modified IA are 

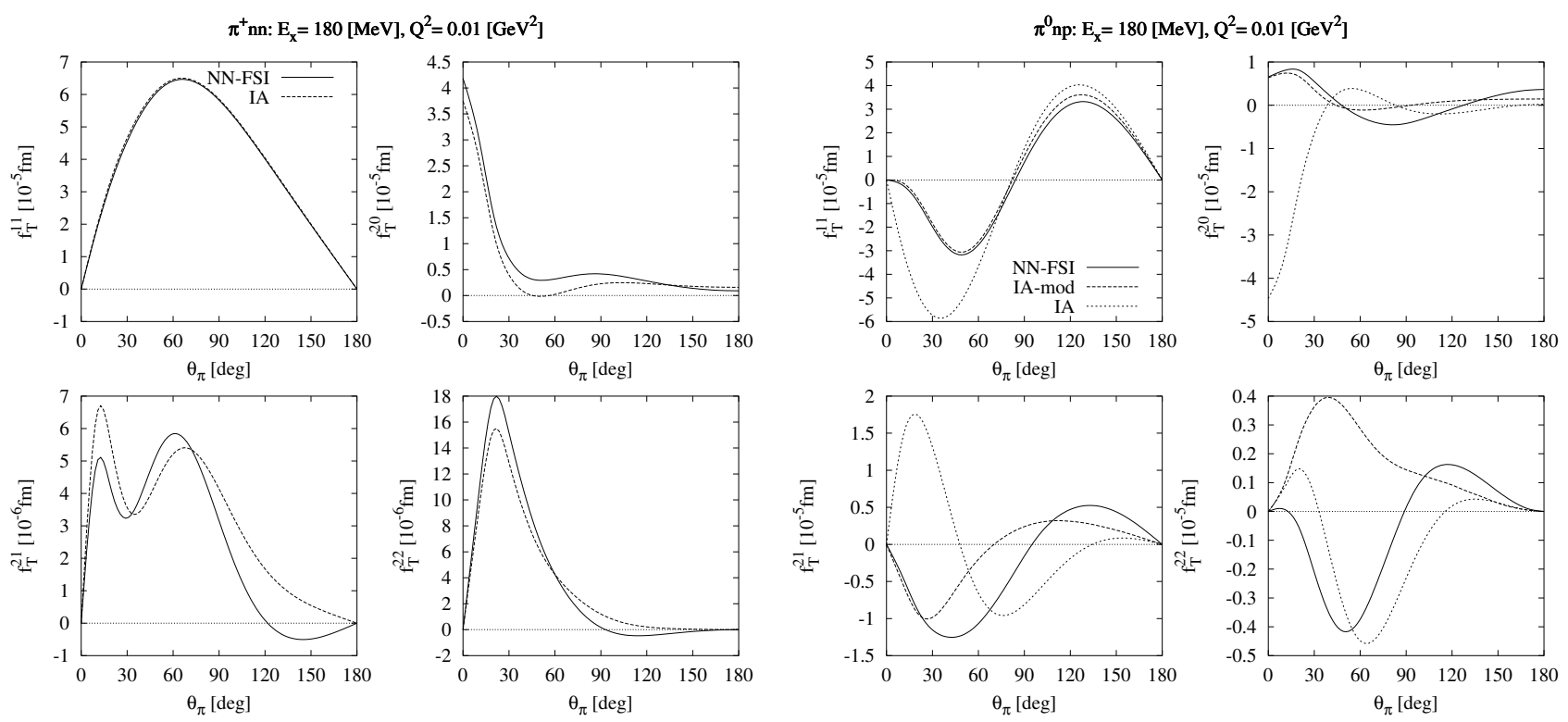

FIG. 8: Polarized transverse structure functions for $\pi^{+}$(left four panels) and $\pi^{0}$ electroproduction (right four panels) at excitation energy $E_{x}=180 \mathrm{MeV}$ and squared four momentum transfer $Q^{2}=0.01 \mathrm{GeV}^{2}$ with $N N$-rescattering in the final state (NN-FSI) and without (IA). For $\pi^{0}$ production results for the modified IA are also given.


FIG. 9: Selected polarized longitudinal-transverse interference structure functions for $\pi^{+}$(left four panels) and $\pi^{0}$ electroproduction (right four panels) at excitation energy $E_{x}=180 \mathrm{MeV}$ and squared four momentum transfer $Q^{2}=0.01 \mathrm{GeV}^{2}$ with $N N$-rescattering in the final state (NN-FSI) and without (IA). For $\pi^{0}$ production results for the modified IA are also given.

small in $f_{T}^{11}$ and $f_{T}^{20}$, but more pronounced in the other structure functions of smaller absolute size, in particular very strong in the smallest $f_{T}^{22}$.

Of the eight polarized $L T$-interference structure functions we show in Fig. 9 the four most dominant ones which are comparable in size to the unpolarized ones. They show quite different characteristic angular behavior. In particular for $\pi^{+}$production, $f_{L T}^{11}$ and $f_{L T}^{21}$ possess a sizeable peak at $0^{\circ}$. All structure functions are almost independent from FSI for $\pi^{+}$production, and even for $\pi^{0}$ production they exhibit little FSI effects beyond the modified IA.

Also of the remaining polarized structure functions of $T T$-type and for longitudinally polarized electrons only a few are displayed in Fig. 10] Again we note very little influence of $N N$-rescattering for both $\pi^{+}$production as well as for $\pi^{0}$ production beyond the modified IA. Of particular interest is $f_{T}^{\prime 10}$ which determines the contribution of single pion production on the deuteron to the generalized Gerasimov-Drell-Hearn sum rule [17]. To conclude this survey, we 

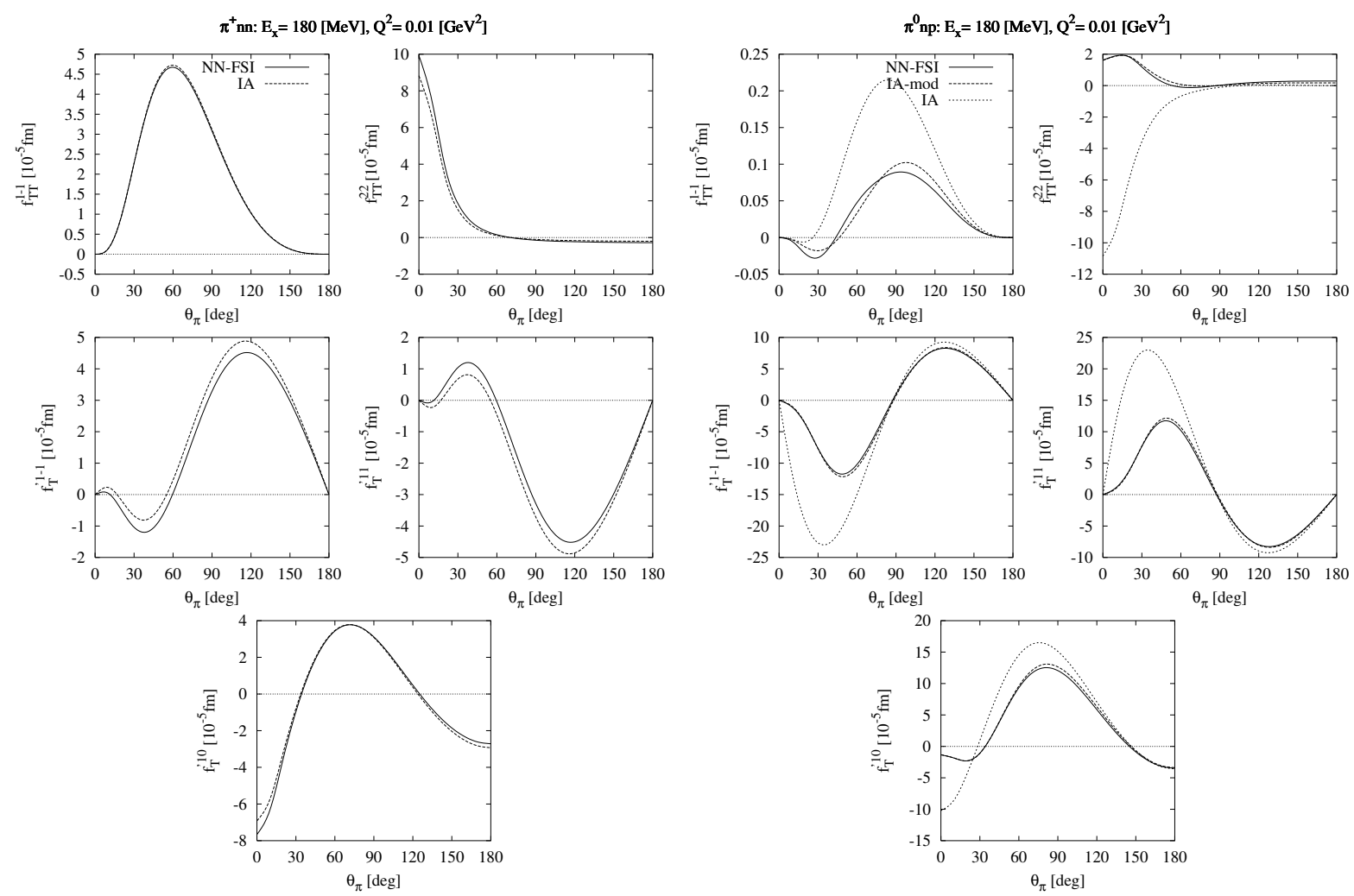

FIG. 10: Selected polarized structure functions of type $T T$ and for polarized electrons of type $T$ for $\pi^{+}$(left five panels) and $\pi^{0}$ electroproduction (right five panels) at excitation energy $E_{x}=180 \mathrm{MeV}$ and squared four momentum transfer $Q^{2}=0.01 \mathrm{GeV}^{2}$ with $N N$-rescattering in the final state (NN-FSI) and without (IA). For $\pi^{0}$ production results for the modified IA are also given.

show in Fig. 11] the $Q^{2}$-dependence of some selected examples of polarized structure functions.

\section{B. Comparison with other calculations and experiment}

We will begin the comparison with an earlier calculation by Loucks et al. [7] who have studied the influence of FSI for the kinematics of the Saclay experiment [5] using a simple pion production model. In particular, they found a strong dependence of the semi-exclusive cross section on the deuteron orientation by considering the idealized case of an intial deuteron state being prepared in a state with definite spin projection $m_{d}^{0}$ on the momentum transfer. This means in our formalism deuteron orientation angles $\theta_{d}=0$ and $\phi_{d}=0$ and a deuteron density matrix $\rho_{m_{d}}^{d} m_{d^{\prime}}=\delta_{m_{d} m_{d^{\prime}}} \delta_{m_{d} m_{d}^{0}}$. The corresponding vector and tensor orientation parameters $P_{1}^{d}$ and $P_{2}^{d}$, respectively, as function of $m_{d}^{0}$ are obtained from (35). Specifically, one finds

$$
\begin{array}{lll}
m_{d}^{0}=0: & P_{1}^{d}(0)=0, & P_{2}^{d}(0)=-\sqrt{2}, \\
m_{d}^{0}= \pm 1: & P_{1}^{d}( \pm 1)= \pm \frac{3}{\sqrt{2}}, & P_{2}^{d}( \pm 1)=\frac{1}{\sqrt{2}} .
\end{array}
$$

In fact, these two values for $P_{2}^{d}$ mark the maximal and minimal possible values for the tensor polarization. For these two cases, one finds from (54) for pion emission along $\vec{q}$

$$
\begin{aligned}
\left.\frac{d^{6} \sigma\left(\Omega_{\pi}=(0,0), \Omega_{d}=(0,0)\right)}{d E_{e}^{\prime} d \Omega_{e}^{\prime} d p_{\pi} d \Omega_{\pi}}\right|_{m_{d}=0} & =\frac{\alpha_{q e d}}{Q^{4}} \frac{k_{e}^{\prime}}{k_{e}}\left[\rho_{L} \widetilde{f}_{L}^{00}+\rho_{T} \widetilde{f}_{T}^{00}-\sqrt{2}\left(\rho_{L} \widetilde{f}_{L}^{20}+\rho_{T} \widetilde{f}_{T}^{20}\right)\right], \\
\left.\frac{d^{6} \sigma\left(\Omega_{\pi}=(0,0), \Omega_{d}=(0,0)\right)}{d E_{e}^{\prime} d \Omega_{e}^{\prime} d p_{\pi} d \Omega_{\pi}}\right|_{m_{d}= \pm 1} & =\frac{\alpha_{q e d}}{Q^{4}} \frac{k_{e}^{\prime}}{k_{e}}\left[\rho_{L} \widetilde{f}_{L}^{00}+\rho_{T} \widetilde{f}_{T}^{00}+\frac{1}{\sqrt{2}}\left(\rho_{L} \widetilde{f}_{L}^{20}+\rho_{T} \widetilde{f}_{T}^{20}\right)\right] .
\end{aligned}
$$




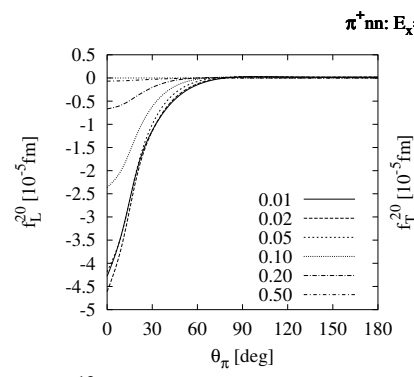

$\mathrm{E}_{\mathrm{x}}=180[\mathrm{MeV}]$
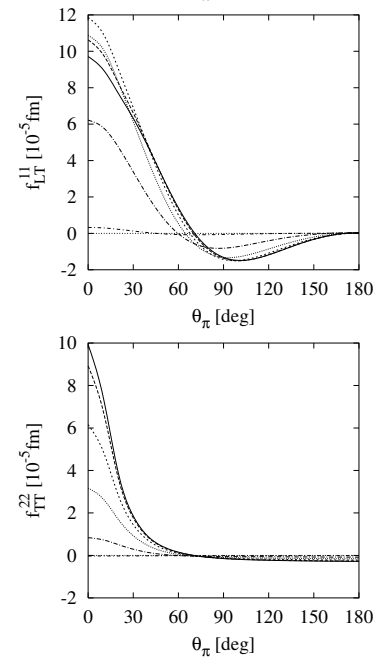


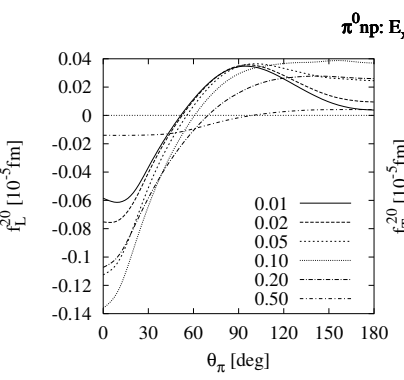

$\pi^{0} \mathrm{np}: \mathrm{E}_{\mathrm{x}}=180[\mathrm{MeV}]$
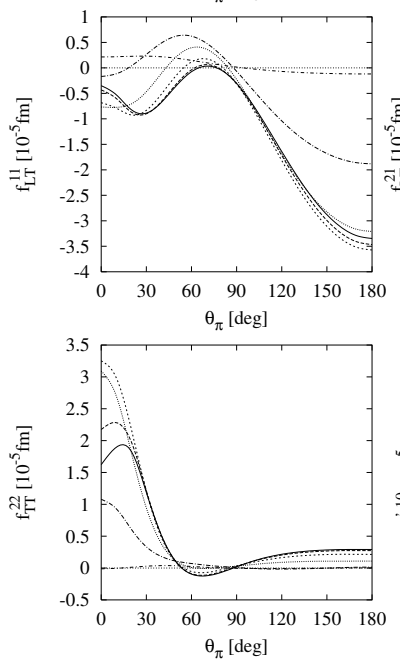
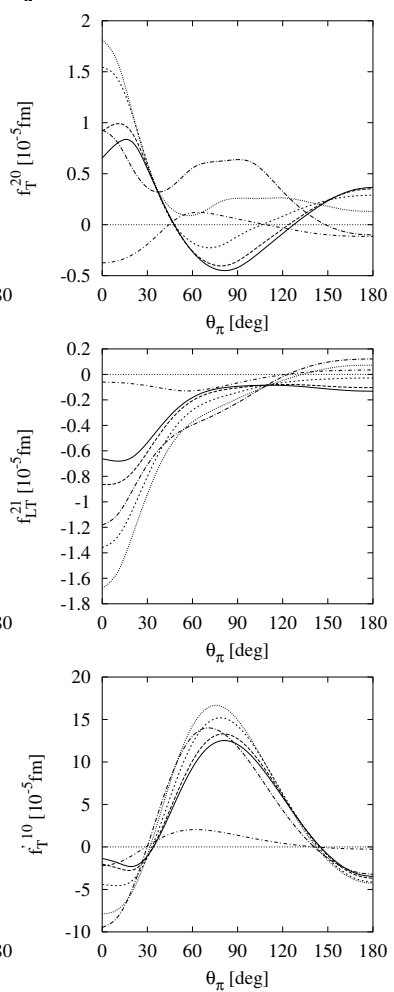

FIG. 11: $Q^{2}$-dependence of selected polarization structure functions of $\pi^{+}$(left six panels) and $\pi^{0}$ (right six panels) electroproduction at excitation energy $E_{x}=180 \mathrm{MeV}$ and various squared four momentum transfers $Q^{2}=0.01,0.02,0.05,0.1,0.2$, and $0.5 \mathrm{GeV}^{2}$.
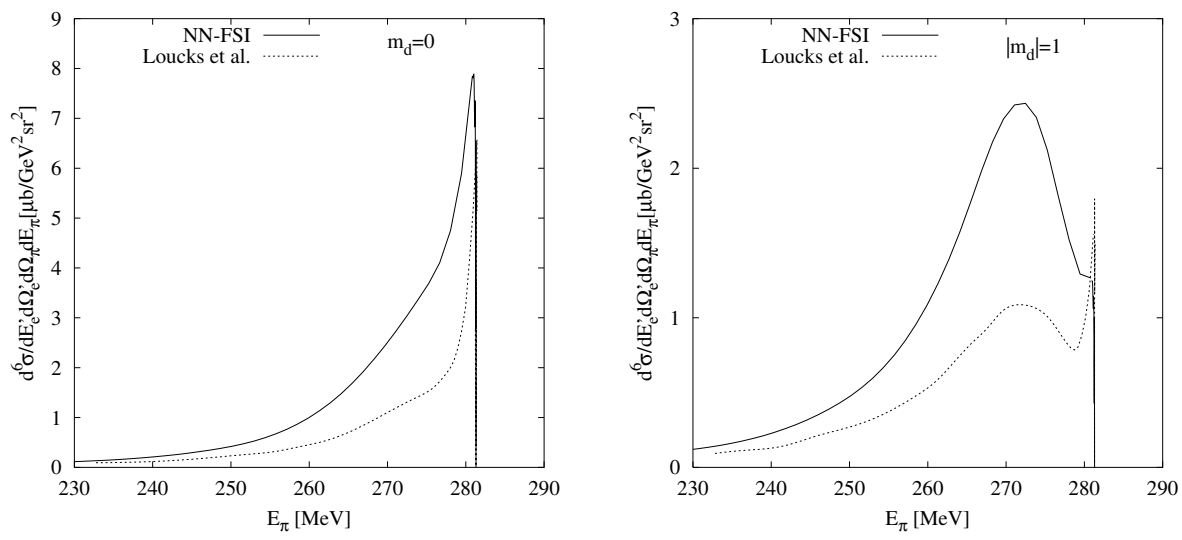

FIG. 12: Semi-exclusive differential cross sections for $\pi^{+}$production on a deuteron with definite spin projection $m_{d}=0$ (left panel) and $\left|m_{d}\right|=1$ (right panel) at $W=2126 \mathrm{MeV}$ for the kinematics of the Saclay experiment 5] ( $E_{e}=645 \mathrm{MeV}$, $E_{e^{\prime}}=355 \mathrm{MeV}, \theta_{e}=36^{\circ}$ ). Notation: solid curves: present results; dotted curves: results of Loucks et al. [7] divided by four.

We compare in Figs. [12 and [13] the results of [7] with the present calculation. In view of the fact, that the elementary production model of [7] gives a cross section too large by about a factor four compared to experiment as pointed out in [9], we have renormalized the results of [7] by this factor. Qualitatively, we find the same dependence on the orientation of the deuteron, namely, for $m_{d}=0$ a dominant contribution from the antibound ${ }^{1} S_{0}-N N$-state near the $N N$-threshold at $W=2126 \mathrm{MeV}$ masking completely the quasi-free peak which is located at $E_{\pi}^{q f, l a b}=271.8 \mathrm{MeV}$ according to (8) (left panel of Fig. 12), whereas for $\left|m_{d}\right|=1$ one notes a strong suppression of the ${ }^{1} S_{0}$-state at the same invarient energy $W$ so that the quasi-free peak becomes clearly seen (right panel of Fig. 12). At the higher energy $W=2205 \mathrm{MeV}$ in Fig. [13 the dependence on the orientation is much weaker. For both orientations the ${ }^{1} S_{0}$-peak 

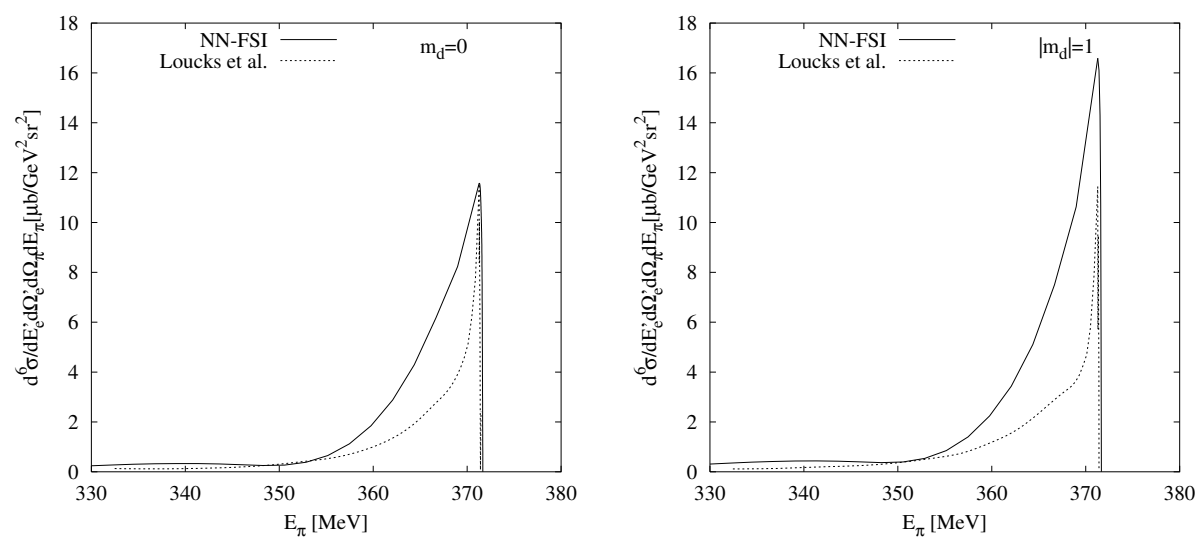

FIG. 13: Same as in Fig. [12 at $W=2205 \mathrm{MeV}$ for the kinematics of the Saclay experiment [5] $\left(E_{e}=645 \mathrm{MeV}, E_{e^{\prime}}=269 \mathrm{MeV}\right.$, $\left.\theta_{e}=36^{\circ}\right)$.

is the dominant feature, and the quasi-free peak at $E_{\pi}^{q f, l a b}=340.5 \mathrm{MeV}$ is barely seen. However, on a quantitative level, we find quite significant differences to the results of [] apart from the overall strength. At $W=2126 \mathrm{MeV}$, we obtain a much stronger suppression of the ${ }^{1} S_{0}$-state appearing only as a shoulder. Furthermore, at lower pion energies, i.e. with increasing $N N$-energy, we find significantly higher strength near the quasi-free peak by almost a factor two. The origin of this difference is not clear. Also at the higher energy $W=2205 \mathrm{MeV}$ one notes again much higher strengths at lower pion energies and moreover also a slightly stronger dependence on the tensor polarization. Now we turn to a comparison of our results with experimental data and with the calculations of Hafidi and Lee [8]


FIG. 14: Semi-exclusive differential cross sections for $d\left(e, e^{\prime} \pi^{+}\right)$in parallel kinematics, i.e. pion emission along the momentum transfer as functions of the missing mass $M_{x}$. Left panel: kinematics of Saclay data [5] $\left(E_{e}=645 \mathrm{MeV}, E_{e}^{\prime}=355 \mathrm{MeV}\right.$, $\left.\theta_{e}^{\prime}=36^{\circ}\right)$; Right panel: kinematics of Jefferson Lab data [6] $\left(E_{e}=845 \mathrm{MeV}, E_{e}^{\prime}=395 \mathrm{MeV}, Q^{2}=0.4 \mathrm{GeV}^{2}\right)$. Notation of curves: solid: present calculations with NN-FSI included; dashed: IA alone; dash-dot: calculations of Hafidi and Lee [8]; dotted: calculations of Levchuk et al. [9].

and Levchuk et al. 9] in Fig. 14] The left panel shows the semi-exclusive differential cross section for pion emission in forward direction for the kinematics of the Saclay data [5] while the right panel shows the one for the kinematics of the Jefferson Lab data [6].

For the Saclay data (left panel of Fig. 114) all three calculations give very similar results for the maximum of the data, which coincides with the position of the quasi-free kinematics, i.e. $M_{x}^{q f}=1885 \mathrm{MeV}$, and at higher missing mass all of them slightly underestimate the data. However, at lower missing mass our calculation as well as the one of Levchuk et al. exhibit the sharp and very pronounced ${ }^{1} S_{0}$-peak right at threshold which is absent in the calculation of Hafidi and Lee and also not seen in the data. It remains a puzzle why this pronounced peak, which is seen in deuteron electrodisintegration near threshold 21] as well as in pion photoproduction 22], is absent in the data of 5].

In contrast to this, the three theoretical results for the kinematics of the Jefferson Lab data (right panel of Fig. 141) differ substantially in the quasi-free maximum $\left(M_{x}^{q f}=1931 \mathrm{MeV}\right)$. While the results of Levchuk et al. overshoot the 
maximum, the one of Hafidi and Lee underestimate it by about the same amount. In this case, our calculation gives a fairly good account of the data although one still notes a slight systematic underestimation. Near threshold we and also Levchuk et al. find again a sharp ${ }^{1} S_{0}$-peak but differing in height while in the results of Hafidi and Lee there appears no indication of such a peak at all.

\section{CONCLUSION AND OUTLOOK}

In the present paper we have investigated the influence of final state interactions in pion electroproduction on the deuteron for energies from threshold up to the second resonance region and squared momentum transfers between $0.01 \mathrm{GeV}^{2}$ and $0.5 \mathrm{GeV}^{2}$. Special emphasis was laid on the study of polarization observables for beam and target polarization. Formal expressions for all structure functions as quadratic hermitean forms in the production amplitudes were derived which govern the unpolarized differential cross section as well as the various polarization observables extending thus the formal developments for pion photoproduction in [1].

The semi-exclusive structure functions of $d\left(e, e^{\prime} \pi\right) N N$ were then evaluated taking the elementary operator for $e N \rightarrow e^{\prime} \pi N^{\prime}$ from the MAID-2003 analysis. The interaction in the final state was included on the basis of the two-body $t$-matrices for $N N$ and $\pi N$ scattering, respectively, restricting their contribution to the first order in the multiple scattering series. With respect to the energy region above the $\Delta(1232)$ resonance, the present work represents the first realistic calculation which extends into the second resonance region. The results show that primarily the $N N$-interaction is important leading to sizeable modifications of some of the structure functions, especially in the energy region up to the $\Delta$ resonance. On the other hand, the effect of pion rescattering is significant and should be included only close to threshold, much below the $\Delta$ resonance. At higher energies it can safely be neglected.

Most visibly distorted by the interaction between the final particles are the unpolarized as well as polarized structure functions in the $\pi^{0}$ channel in contrast to the $\pi^{+}$channel which is much less sensitive to FSI effects. As in the case of photoproduction, this strong effect in the $\pi^{0}$ channel is almost completely due to a spurious contribution of the coherent reaction because of the non-orthogonality of the deuteron wave function to the plane wave of the final two nucleons in IA. After removing this contribution, the remaining FSI effect is similar in size to what has been found in the charged channel.

With respect to the few existing experimental data we find in general a satisfactory agreement with the data for the missing mass spectra of $d\left(e, e^{\prime} \pi^{+}\right)$in parallel kinematics measured at Saclay [5] and JLab [ $[\underline{]}$ around the quasi-free peaks. In comparison to the theoretical results of [] we find a similar strong dependence on the tensor polarization of an oriented deuteron target in the near threshold region. However, we obtain a much stronger quasi-free peak relative to the ${ }^{1} S_{0}$ spike right at threshold. With respect to the work of [8] we see a much stronger influence of the $N N$ interaction at very low excitation energies in the $N N$ subsystem as manifest by the ${ }^{1} S_{0}$ peak which is not present in the results of [8]. On the other hand, we do not find this peak so pronounced as predicted in $[9]$. The origin of these differences is not clear.

In view of the fact that at present only few data are available for the unpolarized semi-exclusive differential cross section of $\pi^{+}$electroproduction at very forward angles, there is an urgent need for more measurements of angular distributions at higher energies and for various momentum transfers for all three charge states of single pion electroproduction on the deuteron. Furthermore, polarization data are totally missing, although they would provide a more detailed analysis of this reaction, in particular, a more detailed investigation of the elementary reaction on the neutron in $\pi^{-}$production on the deuteron. It is therefore very desirable to have new precise experiments in order to improve our knowledge of pion electroproduction on nucleon and deuteron.

\section{APPENDIX: RELATION TO OTHER FORMAL EXPRESSIONS}

In this appendix we would like to give the relations of the structure functions $\widetilde{f}_{\alpha}^{00}$ defined in (55) to other ones used in the literature. We begin with the expression for the semi-exclusive differential cross section of Loucks et al. [7]

$$
\frac{d^{6} \sigma}{d E_{e^{\prime}} d \Omega_{e^{\prime}} d E_{\pi} d \Omega_{\pi}}=\sigma_{M} \frac{p_{\pi} E_{\pi} M p}{12(2 \pi)^{3}}\left[v_{L} R_{L}+v_{T} R_{T}+v_{L T} R_{L T}-v_{T T} R_{T T}\right],
$$

where we assume that all quantities refer to the lab system although this is not stated explicitly in [7]. In fact, in this appendix all variables refer to the lab system if not stated otherwise and indicated specifically. Here, the Mott cross section is denoted by $\sigma_{M}=\alpha_{q e d}^{2} \cos ^{2}\left(\theta_{e} / 2\right) / 4 E_{e}^{2} \sin ^{4}\left(\theta_{e} / 2\right)$, and the kinematic functions $v_{\alpha}$ with $\alpha \in\{L, T, L T, T T\}$ are related to the virtual photon density matrix in (29) by

$$
v_{\alpha}=\frac{2 \eta}{Q^{2}} \beta^{-g_{\alpha}}(\sqrt{2})^{\delta_{\alpha, L T}}(-)^{\delta_{\alpha, T T}} \rho_{\alpha},
$$


where we have introduced for convenience

$$
g_{\alpha}=2 \delta_{\alpha, L}+\delta_{\alpha, L T} .
$$

Comparing (A1) with (54), one finds the following relation

$$
\begin{aligned}
R_{\alpha}\left(E_{\pi}, \Omega_{\pi}\right)= & \left(-\frac{1}{\sqrt{2}} \cos \phi_{\pi}^{c . m .}\right)^{\delta_{\alpha, L T}}\left(-\cos 2 \phi_{\pi}^{c . m .}\right)^{\delta_{\alpha, T T}} \beta^{g_{\alpha}} \frac{6(2 \pi)^{3}}{\alpha_{q e d} M p p_{\pi}^{2}} \\
& \times J\left(p_{\pi}^{c . m .}, \Omega_{\pi}^{c . m .} ; E_{\pi}, \Omega_{\pi}\right) \widetilde{f}_{\alpha}^{00}\left(p_{\pi}^{c . m .}, \theta_{\pi}^{c . m .}\right),
\end{aligned}
$$

where the Jacobian

$$
J\left(p_{\pi}^{c . m .}, \Omega_{\pi}^{c . m .} ; E_{\pi}, \Omega_{\pi}\right)=\left|\frac{\partial\left(p_{\pi}^{c . m .}, \Omega_{\pi}^{c . m .}\right)}{\partial\left(E_{\pi}, \Omega_{\pi}\right)}\right|=\frac{p_{\pi} E_{\pi}^{c . m .}}{\left(p_{\pi}^{c . m .}\right)^{2}}
$$

takes care of the transformation from the c.m. frame variables to the lab frame ones, because we had defined the structure functions $f_{\alpha}^{00}$ with respect to c.m. variables.

As next we consider the expression for the semi-exclusive differential cross section of Levchuk et al. 9]

$$
\frac{d^{6} \sigma}{d E_{e^{\prime}} d \Omega_{e^{\prime}} d E_{\pi} d \Omega_{\pi}}=\sigma_{M} p_{\pi} E_{\pi}\left[\xi^{2} W_{C}+\left(\eta+\frac{\xi}{2}\right) W_{T}-\xi \sqrt{\eta+\xi} \cos \phi_{\pi} W_{I}+\frac{\xi}{2} \cos 2 \phi_{\pi} W_{S}\right],
$$

where $\eta$ and $\xi$ are given in (32). Noting the relations to the virtual photon density matrix in (29)

$$
\xi^{2}=\frac{1}{\beta^{2}} \frac{2 \eta}{Q^{2}} \rho_{L}, \quad \eta+\frac{\xi}{2}=\frac{2 \eta}{Q^{2}} \rho_{T}, \quad \xi \sqrt{\eta+\xi}=\frac{\sqrt{2}}{\beta} \frac{2 \eta}{Q^{2}} \rho_{L T}, \quad \frac{\xi}{2}=-\frac{2 \eta}{Q^{2}} \rho_{T T}
$$

with $\beta$ also given in (32), and changing slightly the notation by setting $W_{L}=W_{C}$ and $W_{L T}=W_{I}$, one finds as relation

$$
W_{\alpha}\left(E_{\pi}, \theta_{\pi}\right)=(-)^{\delta_{\alpha, T T}}\left(-\frac{1}{\sqrt{2}}\right)^{\delta_{\alpha, L T}} \frac{\beta^{g_{\alpha}}}{2 \alpha_{q e d} p_{\pi}^{2}} J\left(p_{\pi}^{c . m .}, \Omega_{\pi}^{c . m .} ; E_{\pi}, \Omega_{\pi}\right) \widetilde{f}_{\alpha}^{00}\left(p_{\pi}^{c . m .}, \theta_{\pi}^{c . m .}\right) .
$$

Finally, we will consider the parametrization in terms of a virtual photon flux times a virtual photon cross section as, for example, used in [6, 8], i.e.

$$
\frac{d^{6} \sigma}{d E_{e^{\prime}} d \Omega_{e^{\prime}} d M_{x} d \Omega_{\pi}}=\Gamma \frac{d^{3} \sigma_{v}}{d M_{x} d \Omega_{\pi}}
$$

where $M_{x}=\sqrt{W^{2}+m_{\pi}^{2}-2 W E_{\pi}^{c . m} \text {. }}$ denotes the missing mass. The virtual photon flux $\Gamma$ is defined by

$$
\Gamma=\frac{\alpha_{q e d}}{2 \pi^{2}} \frac{E_{e^{\prime}}}{E_{e}} \frac{K}{Q^{2}} \frac{1}{1-\varepsilon},
$$

where $K=\left(W^{2}-M_{d}^{2}\right) / 2 M_{d}$ in [8] and $K=\left(W^{2}-M^{2}\right) / 2 M$ in [6] , and the virtual photon cross section by

$$
\frac{d^{3} \sigma_{v}}{d M_{x} d \Omega_{\pi}}=\frac{d^{3} \sigma_{T}}{d M_{x} d \Omega_{\pi}}+\varepsilon \frac{d^{3} \sigma_{L}}{d M_{x} d \Omega_{\pi}}+\sqrt{2 \varepsilon(1+\varepsilon)} \frac{d^{3} \sigma_{L T}}{d M_{x} d \Omega_{\pi}} \cos \phi_{\pi}+\varepsilon \frac{d^{3} \sigma_{T T}}{d M_{x} d \Omega_{\pi}} \cos 2 \phi_{\pi},
$$

where $\varepsilon=\xi /(\xi+2 \eta)$. In $[\underline{8}]$ only the first two terms were included since only pion emission along $\vec{q}$ was considered where the the two interference terms vanish. Using the relations

$$
\frac{1}{1-\varepsilon}=\frac{2}{Q^{2}} \rho_{T}, \quad \varepsilon=-\frac{\rho_{T T}}{\rho_{T}}=\frac{1}{2 \beta^{2} \xi} \frac{\rho_{L}}{\rho_{T}}, \quad \sqrt{2 \varepsilon(1+\varepsilon)}=\sqrt{\frac{2}{\beta^{2} \xi}} \frac{\rho_{L T}}{\rho_{T}},
$$

one finds for the cross section a form analogous to our expression in (54)

$$
\begin{aligned}
\frac{d^{6} \sigma}{d E_{e^{\prime}} d \Omega_{e^{\prime}} d M_{x} d \Omega_{\pi}}= & \frac{\alpha_{q e d}}{\pi^{2}} \frac{E_{e^{\prime}}}{E_{e}} \frac{K}{Q^{4}}\left[\frac{1}{2 \beta^{2} \xi} \rho_{L} \frac{d^{3} \sigma_{L}}{d M_{x} d \Omega_{\pi}}+\rho_{T} \frac{d^{3} \sigma_{T}}{d M_{x} d \Omega_{\pi}}\right. \\
& \left.+\sqrt{\frac{2}{\xi}} \frac{1}{\beta} \rho_{L T} \frac{d^{3} \sigma_{L T}}{d M_{x} d \Omega_{\pi}} \cos \phi_{\pi}-\rho_{T T} \frac{d^{3} \sigma_{T T}}{d M_{x} d \Omega_{\pi}} \cos 2 \phi_{\pi}\right] .
\end{aligned}
$$


Comparison of this expression with (154) yields as final relation

$$
\frac{d^{3} \sigma_{\alpha}}{d M_{x} d \Omega_{\pi}}=\frac{(\beta \sqrt{2 \xi})^{g_{\alpha}}}{2^{\delta_{\alpha, L T}}} \frac{\pi^{2}}{K} J\left(p_{\pi}^{c . m .}, \Omega_{\pi}^{c . m} ; M_{x}, \Omega_{\pi}\right) \widetilde{f}_{\alpha}^{00}\left(p_{\pi}^{c . m}, \theta_{\pi}^{c . m \cdot}\right) .
$$

with

$$
J\left(p_{\pi}^{c . m .}, \Omega_{\pi}^{c . m \cdot} ; M_{x}, \Omega_{\pi}\right)=\left(\frac{p_{\pi}}{p_{\pi}^{c . m .}}\right)^{2} \frac{E_{\pi}^{c . m \cdot} \cdot M_{x}}{\left|\left(q_{0}+M_{d}\right) p_{\pi}-E_{\pi} q \cos \theta_{\pi}\right|}
$$

[1] H. Arenhövel and A. Fix, Phys. Rev. C 72, 064004 (2005).

[2] A. Fix and H. Arenhövel, Phys. Rev. C 72, 064005 (2005).

[3] D. Drechsel, O. Hanstein, S.S. Kamalov, and L. Tiator, MAID: http://www.kph.uni-mainz.de/de/MAID/

[4] C.N. Brown, C.R. Canizares, W.E. Cooper, A.M. Eisner, G.J. Feldman, C.A. Lichtenstein, L. Litt, W. Lockeretz, V.B. Montana, and F.M. Pipkin, Phys. Rev. Lett. 27, 536 (1971).

[5] R. Gilman et al., Phys. Rev. Lett. 64, 622 (1990).

[6] D. Gaskell et al., Phys. Rev. Lett. 87, 202301 (2001).

[7] R.J. Loucks, V.R. Pandharipande, and R. Schiavilla, Phys. Rev. C 49, 342 (1994).

[8] K. Hafidi and T.-S.H. Lee, Phys. Rev. C 64, 064607 (2001).

[9] L. Levchuk, L. Canton, and A. Shebeko, Eur. Phys. J. A 21, 29 (2004).

[10] H. Arenhövel, Few-Body Syst. 4, 55 (1988).

[11] E.M. Rose, Elementary Theory of Angular Momentum, Wiley, New York 1957.

[12] B.A. Robson, The Theory of Polarization Phenomena, Clarendon Press, Oxford 1974.

[13] H. Arenhövel, W. Leidemann, and E.L. Tomusiak, Eur. Phys. J. A 23, 147 (2005).

[14] T.W. Donnelly and A.S. Raskin, Ann. Phys. (N.Y.) 169, 247 (1986).

[15] J. Haidenbauer and W. Plessas, Phys. Rev. C 30, 1822 (1984); 32, 1424 (1985).

[16] S. Nozawa, B. Blankleider, and T.-S.H. Lee, Nucl. Phys. A 513, 459 (1990).

[17] H. Arenhövel, Phys. Lett. B 595, 223 (2004).

[18] J.-M. Laget, Phys. Rep. 69, 1 (1981).

[19] E. Darwish, H. Arenhövel, and M. Schwamb, Eur. Phys. J. A 16, 111 (2003).

[20] M.I. Levchuk, M. Schumacher, and F. Wissman, nucl-th/0011041

[21] G.G. Simon, F. Borkowski, Ch. Schmitt, V.H Walther, H. Arenhövel, and W. Fabian, Nucl. Phys. A 324, 277 (1979).

[22] G. Köbschall, B. Alberti, H. Jansen, K. Rohrich, C. Schmitt, V.H. Walther, K. Weinand, M. Kobayashi, and H. Arenhövel, Nucl. Phys. A 466, 612 (1987). 